\title{
Ja aber - Korpusbasierte Beobachtungen zu einer pragmatisch beschreibbaren Konstruktion
}

\author{
Sven Staffeldt (Würzburg)
}

\begin{abstract}
In spoken conversation as well as in written texts we can find examples of a construction which has been the object of linguistic research for about 35 years and which has repeatedly been described under the names of ja-aber syntagma, ja-aber connection and ja-aber utterance pattern. In most analyses this construction is split into two parts: The ja-part is seen to convey (partial) consent, whereas the function of the aber-part is interpreted as (partial) objection. Falling back upon $j a$ as affirmative response particle and aber as adversative conjunction, such analyses operate on semantically compositional principles. Additionally, the possibility of $j a$ being a structuring particle is taken into consideration, where $j a$ is supposed to ensure the mere connection of the part following the particle with the antecedent part. However, as this paper wants to show, compositional approaches like these tend to mislead studies to circular lines of arguments, in which presumptive findings are taken for presuppositions (petitio principii). Moreover, they seem to disregard actual usage of this construction, which can be ascertained with the help of corpora. Corpus-aided investigation of the construction in question shows that its semantic elaboration is dependent on two factors that go beyond compositional explanation attempts: (1) syntactical integration of the aber-part and (2) presence of negation in the aberpart. Not even in "classic" examples this construction can be semantically reduced to simple combinations of consent + objection, though. In order to reach appropriate results here, semantic analysis has to turn to pragmatically more adequate ways of description.
\end{abstract}

\section{Das Interesse an ja aber}

\subsection{Lexikologisch-phraseologische Ausgangssituation}

Aus lexikologischer Sicht gehört aber als Konjunktion zu der geschlossenen Klasse von Funktionswörtern, die modular eher syntaktischer als lexikalischer Natur sind, weil sie nicht über eine semantisch-begriffliche Bedeutung verfügen und damit nicht benennen, sondern über eine grammatisch-relationale Bedeutung und damit Beziehungen zwischen Wörtern der offenen Klasse herstellen (cf. Aitchison 1997: 140; Schippan 2002: ; Lutzeier 1995: 34). Aber auch ja als Antwortpartikel ist lexikologisch keine oder keine zentrale Einheit, wiederum wegen der fehlenden begrifflichen Bedeutung. Aus dem gleichen Grund sind ja und aber als Modalpartikeln, auf die wir hier in diesem Aufsatz aber nicht näher eingehen, ebenfalls höchstens periphere Kandidaten für lexikalische Einheiten. Was für die beiden einzelnen Einheiten gilt, müsste dann erst recht für die Verkettung derselben gelten. Ja aber kann als Aufeinanderfolge zweier

Linguistik online 90, 3/18 - http://dx.doi.org/10.13092/lo.90.4319

CC by 3.0 
nicht-lexikalischer Einheiten selbst nur nicht-lexikalisch sein. Innerhalb der Phraseologie zeigt sich ein solcher Standpunkt z. B. in einer etwaigen definitorischen Festlegung, dass Phraseologismen mindestens ein Autosemantikon enthalten müssen (cf. Fleischer ${ }^{2}$ 1997: 82, eine Festlegung, die innerhalb der Phraseologie aber nicht unumstritten ist (cf. Burger ${ }^{4} 2010$ : 15)). Wenn das so ist, bleibt für die Beschreibung des verketteten Vorkommens von ja und aber nur ein kompositionaler Zugriff: $J a$ hat etwas mit positiver Rückmeldung zu tun, mit Zustimmung im weitesten Sinn, und aber leitet etwas Adversatives ein, einen Widerspruch im weitesten Sinn.

\subsection{Trübungen}

Eine erste Trübung erhält diese Perspektive durch das möglicherweise sprachspielerisch kreative, in puncto grammatischer Akzeptabilität aber sicher unauffällige Vorkommen von ja aber als Determinans in Komposita. Beispiele hierfür findet man im Internet schnell und problemlos, so etwa Komposita auf der Seite des Ja-aber ${ }^{\circledR}$-Seminars ${ }^{1}$, die nicht nur auf den Firmennamen zurückführbar sind, z. B.: Ja-aber-Verhalten oder Ja-aber-Denken. Als LeserIn eines solchen Kompositums dürften Sie weder Verständnisschwierigkeiten haben noch das - zugegeben: ungewöhnliche - Kompositum brüsk als morphologisch inakzeptabel (etwa als Fehler) zurückweisen. Sie wissen vermutlich oder können zumindest gute Vermutungen darüber anstellen, was das ist, ein Ja-aber-Denken, auch wenn Sie dieses Wortbildungsprodukt, dessen Modell übrigens auch in der Wortbildungslehre peripher ist oder erhebliche Probleme zu bereiten scheint, ${ }^{2}$ eben zum ersten Mal gesehen haben sollten. Es scheint so, als könne ja aber jedenfalls eine zum Dasein als Bestimmungswort in Determinativkomposita hinreichende Bedeutung annehmen, die sich nicht einfach darauf zurückführen lässt, dass ja aber hier nur erwähnt, aber nicht gebraucht würde. Im Unterschied zu etwa Dass-Satz, wo eine Paraphrase ,Satz, der durch dass eingeleitet wird' die Subjunktion dass als nur erwähnt ausweist, hat Ja-aber in Ja-aberDenken offenbar selbst eine Bedeutung, denn eine Paraphrase der Art, ?,Denken, bei dem man ja aber denkt' oder ?,Denken, das ja aber enthält' o. Ä. wäre wohl als inadäquat anzusehen.

Eine zweite Trübung erfährt diese Perspektive dadurch, dass ja aber offensichtlich als eigenständige Einheit gebraucht werden kann. Das betrifft zum einen Fälle, in denen ja aber als satzäquivalente komplexe Antwortpartikel gebraucht wird: „Stadtwerke? Ja, aber!“3 Als Teil einer Überschrift der Onlineversion der Berliner Zeitung vom 31.01.2013 fasst ,Ja, aber! ‘ hier die Antwort auf die Frage, ob es kommunale Stadtwerke geben wird, zusammen, ohne dass im Text selbst noch einmal ja aber vorkäme. Zum anderen sind damit aber auch Fälle angesprochen, in denen ja aber als Substantivderivat gebraucht vorkommt. Sogar mit zwei harten Kriterien für die Bestimmung als Substantiv, nämlich Artikelfähigkeit und Attribuierbarkeit: „Bleibt ein großes JA - ABER.“4 Auch Belege mit Artikel, linksseitigem Adjektivattribut und rechtsseitigem Relativsatzattribut lassen sich finden, wie hier in diesem Kommentar zu einer PI-Nachricht: „,ein kleines ,ja, aber', das leicht umgangen, mißachtet werden kann“"5.

\footnotetext{
${ }^{1} \mathrm{Cf}$. http://www.ja-aber.de/willkommen. [13.02.2013]

${ }^{2}$ Cf. Fleischer/Barz ${ }^{4}$ 2012: 166: „Unberücksichtigt bleiben solche mit Konjunktionen (Dass-Satz), Interjektion (Aha-Erlebnis) und Satzäquivalent (Jawort).“

${ }^{3}$ Cf. http://www.berliner-zeitung.de/berlin/volksbegehren-energiepolitik-stadtwerke--ja--aber-,10809148,21110 766.html. [13.02.2013]

${ }^{4}$ Cf. http://www.l-talk.de/politiken/ja-aber-erbschaftsteuer-in-der-lebenspartnerschaft.html. [13.02.2013]

${ }^{5}$ Cf. http://www.pi-news.net/2012/04/daubler-gmelin-mit-euro-verfassungsklage. [13.02.2013]
}

ISSN 1615-3014 
Diese Trübungen sind erste Hinweise darauf, dass eine lexikologische Wegwischhaltung gegenüber einer Einheit wie ja aber jedenfalls an einer Gebrauchsrealität vorbeigeht, die diese Verkettung deutlich als bedeutungshaltig und eben nicht nur rein verknüpfend oder relational ausweist. Es wird (auch) hier die Fragestellung deutlich, ob die den Differenzen Autosemantikon vs. Synsemantikon, Inhaltswort vs. Funktionswort, lexikalisch vs. grammatisch zugrundeliegende Abgrenzung von Lexikon und Syntax in dieser Striktheit aufrechterhalten werden kann. Insofern reiht sich diese Untersuchung zu ja aber ein in eine Menge von Studien zu sprachlichen Einheiten, die Anlass dazu geben, über eine strikte Trennung von Lexikon und Syntax neu nachzudenken.

\section{3 ja aber konstruktionsgrammatisch betrachtet}

Wenn ein rein kompositionales Verständnis von ja aber für die Beschreibung der Verwendungsbreite von ja aber kein adäquates Mittel ist, kann daran gedacht werden, ja aber als Konstruktion zu modellieren, im Sinne der Berkeley Construction Grammar (cf. Ziem/Lasch 2013: 50-56) (allerdings ohne dabei deren „unifikationsbasiertes Notationssystem“ (ebd., 53) für die Zwecke der Beschreibung übernehmen zu wollen). Man hätte es dann mit einer idiomatischen Konstruktion zu tun, die (nach den Unterscheidungen von Fillmore/Kay/O'Connor 1988) über die folgenden Eigenschaften verfügt (wie im Einzelnen unten in den Abschnitten 3 und 4 noch zu zeigen sein wird):

- $\quad$ Sie ist dekodierend (decoding idiom): Zwar gibt es eine kompositionale Lesart, man kann $j a$ und aber wörtlich verstehen. Aber für die angenommene Konstruktion wäre wesentlich, dass es darüber hinaus Bedeutungsaspekte der ja-aber-Konstruktion gibt, die sich nicht allein oder nicht vollständig aus dem wörtlichen Verständnis von ja + aber ergeben.

- Sie ist außergrammatisch (extragrammatical idiom): In einem engen Verständnis von Grammatikalität ist die ja aber-Konstruktion keine, die mit einer regelbasierten Syntax adäquat beschrieben werden kann. Das liegt vor allem an dem syntaktisch schwierig zu rekonstruierenden Vorkommen von ja. Ließe sich der aber-Teil allein gut als Koordinierung (auf Wort-, Wortgruppen- oder Satzebene) beschreiben, so kann für ja nicht gleichermaßen eine syntaktisch integrierende Beschreibung angegeben werden.

- Sie ist formal (formal or lexically open idiom): Zwar kann die Hauptfunktion von ja aber in verschiedenen Arten der Fügung sprachlicher Einheiten gesehen und damit als fixe grammatische Bedeutung angenommen werden. Aber die Bedeutung der Konstruktion geht darüber hinaus. Sie ist abhängig davon, welche Einheiten wie gefügt werden, wobei gerade hier - wenn auch eher abstrakt-kategorielle - Verfestigungen einen konstruktionalen Status der gesamten Einheit mit sich bringen. So macht es, wie wir sehen werden, etwa einen Unterschied, ob der durch aber gefügte Teil im weitesten Sinn als syntaktisch integrierbar in den Teil vor $j a$ verstanden werden kann oder nicht. Um die Ergebnisse hier schon einmal vorwegnehmend anzudeuten: Im Falle der syntaktischen Integrierbarkeit liegt die besondere Leistung der ja aber-Konstruktion in dem Splitting eines gesetzten Themas, das selbst nicht als geteiltes eingeführt wurde, und in der Stellungnahme zu diesem Thema. Im Falle der Nicht-Integrierbarkeit hat die ja aber-Konstruktion einen kommunikativ responsiven Status, der nicht mit der splittenden Meinungskundgabe zu einem bestimmten Thema 
zu erfassen ist, sondern in der propositionalen Erweiterung und damit auch illokutionären Reaktion besteht (Hinzufügung).

- Sie hat eine pragmatische Funktion (idiom with pragmatic point): Beim Splitting wird zu einem Thema in seinen Teilaspekten Stellung bezogen, bei der Hinzufügung ein responsivreaktiver Sprechakt vollzogen.

Zur Beschreibung dieser Konstruktion gehört so auch ganz wesentlich der kotextuelle Einbezug des Teils links von oder vor $j a$ und rechts von oder nach aber. Insofern ergibt sich als maximal schematisches Grundgerüst, auf dessen grafische Ausgestaltung im weiteren Text dann aber verzichtet wird, das folgende:

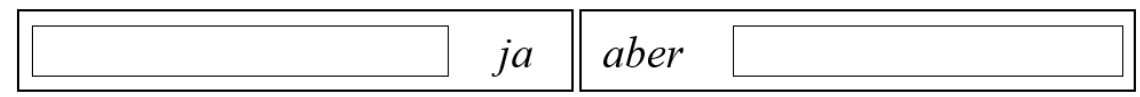

Abbildung 1: Grundgerüst zur Modellierung einer ja-aber-Konstruktion

Der ja-Teil besteht aus dem Teil vor ja + ja selbst, der aber-Teil aus dem Teil nach aber und aber selbst. Sowohl der Teil vor ja als auch der Teil nach aber können dabei unbesetzt bleiben. Bei dieser Konstruktion gibt es mindestens zwei Unterkonstruktionen: Ist der Teil nach aber in den Teil vor ja integrierbar, haben wir es mit der Splittung-Unterkonstruktion zu tun. Ein kognitiv-prozessuales Charakteristikum dieser Konstruktion ist nun, dass der Teil vor ja in seiner Erstreckung vom Verstehenden oftmals selbst erfasst und bei den Formen des verdeckten Splittings als Gegenstück zum aber-Teil zuallererst rekonstruiert werden muss. Ist der Teil nach aber nicht integrierbar, so handelt es sich um die Hinzufügungs-Unterkonstruktion. Beide Unterkonstruktionen werden in den Abschnitten 3 und 4 näher untersucht.

\section{Bisherige Beschäftigung mit ja aber}

In den folgenden vier Abschnitten (2.1 bis 2.4) werden in aller Kürze grundlegende Bestimmungen der ja-aber-Konstruktion, wie sie seit 1977 in der Literatur zu finden sind, vorgestellt. Vor dem Hintergrund dieser Festlegungen können darauf folgend Einzelanalysen in Studien (Abschnitt 2.5) auf ihren Erkenntniszuwachs befragt werden. Dabei wird deutlich werden, dass ein analysierender Zugriff auf ja aber unter den Vorzeichen wörtlicher Lesarten häufig genug zu methodischen Inkonsequenzen verführt. Es schließen sich zwei Abschnitte (2.6 und 2.7) an, in denen sich die Diskussion auf die Frage nach dem lexikopragmatischen Status von ja aber zuspitzt.

\subsection{Lütten 1977}

Aus sprechakttheoretischer Perspektive beschäftigt sich Jutta Lütten mit den deutschen Partikeln aber, also, auch, bloß, dabei, bei, denn, doch, eben, eigentlich, einfach, einmal, erst, etwa, gerade, halt, ja, mal, nämlich, nich(t), noch, nun, nur, richtig, ruhig, schon, so, sogar, vielleicht und wohl. Für ja stellt sie ganz allgemein fest, dass dessen „Funktion [...] in den Texten gesprochener Sprache fast ausschließlich auf den Bereich des Rekurses auf die gemeinsame Basis beschränkt“ (Lütten 1977: 358) ist und nur selten ,als Affirmationsmorphem“ (ebd.) auftritt.6

\footnotetext{
${ }^{6}$ Auch Opalka (1979) stellt in seinen Analysen übrigens vorwiegend metakommunikative Verwendungen von ja fest, nämlich Anzeige von Redebeginn, von Redefortsetzung (worunter Opalka allerdings auch die responsive Verwendung zu fassen scheint), von Redeunterbrechung und von Redeeinschub, wobei in seinem Transkript einer
} 
Als besondere Fälle diskutiert sie schließlich ein parenthetisches $j a$ und „das Syntagma (JA)ABER.“ (ebd.) Hier hält sie fest:

\section{Die Funktion von (JA)ABER}

Eine charakteristische Verwendungsweise von JA stellt in den Texten die Verbindung (JA)ABER dar. Besonders frequent ist sie in der Textsorte ,Diskussion'. Diese beiden Elemente stehen an sich im Widerspruch zueinander, insofern JA primär affirmative Bedeutung hat, ABER hingegen einen Gegensatz bzw. einen Einwand anzeigt. Der besondere kommunikative Sinn der Einheit (JA)ABER erklärt sich jedoch daraus, daß ein Sprecher mittels JA dem Gesprächspartner zu erkennen gibt, daß er dessen Meinungsäußerung wahrgenommen, verstanden hat und als Meinungsäußerung akzeptiert, ohne daß er allerdings die Meinung als solche akzeptiert; im Gegenteil, er macht durch den Gebrauch von ABER deutlich, daß er anderer Ansicht ist, daß er nicht zustimmt. ABER ist also auf der inhaltlichen Seite des Argumentationszusammenhanges zu nennen, wohingegen JA seinen affirmativen Charakter nicht verloren hat; dieser bezieht sich jedoch nicht auf den sachlichen Gehalt einer Äußerung, sondern auf die Ebene der kommunikativen Geltung und Akzeptabilität. Der Sprecher $\mathrm{S}_{1}$ stimmt dem zu, daß $\mathrm{S}_{2}$ eine vom Argumentationszusammenhang her konsequente und sinnvolle Äußerung getan hat, auf die er eingehen wird.

Die Darstellung von parenthetischem (JA) und dem Syntagma (JA)ABER macht deutlich, in welch hohem Maße JA in Texten gesprochener Sprache den Rang einer kommunikativen ,Parti-

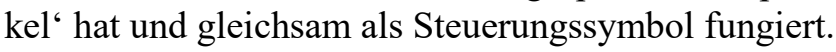

(Lütten 1977: 361)

In ihrer textsortenspezifischen Auswertung (cf. Lütten 1977: 402-611) finden sich allerdings, wenn ich richtig sehe, in den Abschnitten zu ja keine Belege, in denen ja und aber verkettet vorkommen, sondern nur einige der Verkettungen in den Ausführungen zu aber. Dort ist ja allerdings seltsamerweise eingeklammert anzutreffen. Zum Beispiel: ,(ja) ABER ich mein...“. (Lütten 1977: 481) Zu runden Klammern heißt es: „Runde Klammern schließen Erläuterungen zum Kontext oder kurze Paraphrasierungen ein“(Lütten 1977: 401). Trifft das auch hier zu?

Wie wir noch sehen werden, ist eine Funktionsbestimmung auch und gerade von der Strukturbestimmung abhängig: Funktionen hängen nicht in der Luft. Sie bedürfen eines Trägers. Und anders herum: Nur das Vorkommen von sprachlichen Einheiten als Funktionsträgern macht es überhaupt möglich, sprachliche Funktionen analysieren zu können. Was wir brauchen, ist eine strukturelle Beschreibung der Vorkommen von (JA)ABER.

\section{$2.2 \quad$ Koerfer 1979}

Den Anlass, sich mit ja aber zu beschäftigen, beschreibt Koerfer folgendermaßen:

Meine Aufmerksamkeit auf das Phänomen ja aber wurde im Rahmen von Transkriptionen zu einem Projekt zur Hochschulkommunikation erst dadurch gelenkt, daß es in einer bestimmten Sequenz gehäuft auftrat, und zwar bei einem mehrfach mißglückenden Versuch eines spontanen Sprecherwechsels per Selbstwahl. Der aktuelle Sprecher A war jeweils schneller und hartnäckiger mit der Fortführung seines Redebeitrags, während der Sprecher B seinen beabsichtigten Redebeitrag wiederholt mit ja aber einleitete und dann abbrach - weil er eben nicht zum Zuge kam. Ich erwähne diesen besonderen Umstand, durch den ich dann zur weiteren Untersuchung zunächst des Vorkommens von ja aber in Redeeinleitungsposition motiviert wurde, als eines der Argumente für eine Analyse natürlicher, mündlicher Kommunikation.

(Koerfer 1979: 14f.)

einstündigen Diskussion die Anzeige des Redebeginns dominiert: ,,von den ca. 180 vorkommen von einfachem ja gab es 105 verwendungen, die als redesignal in eröffnungsfunktion zu betrachten sind.“ (Opalka 1979: 162). Ja aber findet Opalka in seinem Transkript 5 mal (cf. ebd.).

ISSN 1615-3014 
Aus seinen Transkripten entnimmt Koerfer - allerdings nicht voll authentische, sondern für die Zwecke der Demonstration typisierte - Beispielsequenzen, an denen er die rein kompositionale Lesart mit der affirmativ-adversativen Doppelfunktion unter Beschuss nimmt. Letztere kommt nur in den Fällen infrage, in denen ja tatsächlich als Antwort (z. B. auf eine Entscheidungsfrage) angesehen werden kann und der folgende, durch aber eingeleitete Teil die Zustimmungslesart nicht verunmöglicht. Solche (letztlich doch konstruierten) Belegbeispiele findet Koerfer nun allerdings (cf. Bsp. 10 und 11):

Beispiel (1)

(7) A Ist der Zug schon durch? - B: Ja./Nein.

(8) A Hast du Lorenzer gelesen?

B Ja, aber der bringt uns nicht voran.

(9) A Du unterscheidest hier nicht zwischen, Klischee ' und ,Zeichen“

B Ja, aber das hat gute Gründe.

(10) A Bring mir deine Fußballschuhe mit!

B +7 Ja, aber ich habe keine Fußballschuhe

(11) A Hat dir das Kino gefallen?

B + Ja, aber ich war nicht im Kino.

Koerfer (1979: 21)

In (8) wird mit ja zustimmend auf eine Frage geantwortet und mit aber die, wie Koerfer dies nennt, pragmatische Präsupposition zurückgewiesen, dass „Lorenzer A und B voranbringt“ (Koerfer 1979: 21). In (9) wird mit ja zustimmend auf eine Behauptung reagiert und mit aber die, wie Koerfer dies nennt, semantische Präsupposition zurückgewiesen, „daß das nicht gute Gründe hat“ (ebd.). „Für die Beispiele (10) und (11) gilt diese affirmativ-adversative Doppelfunktion von ja aber nicht. Hier werden jeweils die semantischen Präsuppositionen zurückgewiesen, (10) daß B Fußballschuhe hat und (11) daß B im Kino war.“ (ebd.) Hier könne ja offensichtlich keine affirmative Funktion haben.

Anhand dieser Beispiele kommt Koerfer also zu dem Ergebnis, dass mit ja nicht immer nur Zustimmung signalisiert wird (Akzeptierens-ja), sondern es vielmehr auch ein reines Verstehens-ja gibt, dessen pragmatische Bedeutung in Verbindung mit aber etwa mit ,ja, ich habe dich schon verstanden, aber...' (eigene Paraphrase; d. Verf.) angegeben werden könnte. Die Annahme, „daß dem Komplex ja aber einheitlich eine affirmativ-adversative Doppelfunktion zugeschrieben werden könne“ (Koerfer 1979: 20), kann also nicht aufrecht erhalten werden.

Über diese sequenziell eingebetteten Funktionen hinaus findet Koerfer für ja aber noch zwei weitere wichtige Funktionen. Es trete zum einen auf als themenorientierte Allround-Formel (etwa zur Themenprogression) und zum anderen als personenorientierte Kompromissformel (im Rahmen einer Konfliktvermeidungsstrategie).

$7,,+“=$ hier liegt kein affirmatives $j a$ vor, d. h.: semantisch so lange nicht akzeptabel, wie man für $j a$ nur eine affirmative Funktion annimmt 


\subsection{Kallmeyer 1978, 1987 und 2007}

Einen pragmatisch-onomasiologischen Zugang zur Beschreibung der ja-aber-Konstruktion wählt Kallmeyer. Eine Antwort auf die Variation bestimmter Phänomene sucht er ,auf der Ebene zugrundeliegender Äußerungsmuster [...], die nicht an eine Formulierung mit ,ja aber ‘ gebunden sind, für die diese Formulierung aber vielleicht eine markante Realisierung darstellt" (Kallmeyer 1987: 59). Kallmeyer schließt dabei an frühere Arbeiten an, in denen es um die grundlegende Beschreibung der Prinzipien, Gegenstände und Arbeitsweisen der Konversationsanalyse (Kallmeyer/Schütze 1976) und speziell um Fokuswechsel und Fokussierungen (Kallmeyer 1978) geht. Wichtig für unseren Zusammenhang ist der Pike'sche Fokus-Begriff, mit dem man eine ,Zentriertheit auf einen dominanten Aspekt““ (Kallmeyer/Schütze 1976: 12) bezeichnet:

Dieser Begriff bezieht sich generell auf die menschliche Aufmerksamkeit, die jeweils gerichtet ist und bestimmte Bereiche bzw. Spannen erfaßt [...]. Im Rahmen der Konversationsanalyse sind verschiedene Ebenen der Aufmerksamkeit zu unterscheiden: die Ebene der Gesprächsorganisation, der Handlungsfiguren, der Strategiebildung, übergreifender bürokratisch-institutioneller oder auch biographischer Zusammenhänge usw. Auf der Ebene der Gesprächsorganisation bezeichnet Fokus eine manifestierte, in einem Prozeß des Aushandelns wechselseitig konstituierte bzw. zur Übernahme vorgeschlagene Orientierung. Diese hat eine Selektions- und Hierarchisierungsfunktion: einzelne Themen werden als relevant oder irrelevant, als subsidiär im Hinblick auf übergreifende thematische Gesichtspunkte eingestuft usw.

(Kallmeyer/Schütze 1976: 12)

In diesem Rahmen sieht Kallmeyer auch die Funktionen der ja-aber-Konstruktion:

[E]s gibt in komplexen Äußerungen, unabhängig von der Konstitution von Thema, Fokus usw. im satzgrammatischen Sinn eine Gliederung aufgrund der Relevanzeinstufung. [...] Auf diese Weise wird u. a. eine charakteristische Form eingeleiteter Äußerungen konstituiert: der Typ jaaber, bei dem die Berücksichtigung eines voraufgehend etablierten Fokus (durch die Bestätigung) mit der Abwahl dieses Fokus und der Zuwendung zu einem anderen, konkurrierenden Fokus verbunden wird (aber).

(Kallmeyer 1978: 216)

Werden verschiedene Foki realisiert, ist die funktionale Einheitlichkeit vor allem über die Hierarchisierung der Foki zu gewährleisten. Die häufigsten Muster sind die Einbettung eines untergeordneten Fokus in Form eines Einschubs und die Kombination von einem ersten Teil mit Relevanzrückstufung und einem zweiten Teil mit Relevanzhochstufung. In diesen Äußerungen repräsentiert der zweite Teil das, ,worauf der Sprecher hinauswill' und der Partner reagieren soll, und der erste Teil bearbeitet Dinge, die auch/nebenbei/vorher zu erledigen sind.

(Kallmeyer 1987: 60)

Dieses Äußerungsmuster ist dabei weder auf das Vorkommen von ja aber beschränkt, noch darauf, dass ja und aber kookkurrieren oder gar ausschließlich direkt aufeinander folgen. Vielmehr können verschiedene sprachliche Mittel Äußerungen dieses Typs (mit dieser gesprächsorganisatorischen Funktion) konstituieren. In Kallmeyer $2007^{8}$ finden sich hierfür zahlreiche

\footnotetext{
${ }^{8}$ In diesem Aufsatz beschäftigt sich Kallmeyer mit den „Möglichkeiten der maschinellen Unterstützung bei der Arbeit mit Interaktionskorpora“ (so der Titel). Und zwar vor allem mit der Frage, wie ein variantenreich auftretendes Muster in Korpora gesprochener Sprache (speziell: in der DGD) gesucht werden kann: „Es geht dabei darum, begrenzte Suchmöglichkeiten heuristisch zu nutzen, Grenzen zu reflektieren und Recherchestrategien weiter zu entwickeln und Entwicklungsbedarf zu präzisieren für die Recherche von komplexen Phänomenen, die gegenwärtig kaum erfassbar sind.“ (Kallmeyer 2007: 205) In einem pragmatisch nicht-annotierten Korpus bleiben für die Recherche allerdings lediglich die bekannten Parameter (Suchkette aus Wortformen, logischen Operatoren
} 
Beispiele. Kallmeyer greift seine Beschreibung der ja-aber-Konstruktion als grammatischpragmatisches Muster im Rahmen der Relevanzsetzung wieder auf und nennt zahlreiche sprachliche Einheiten, mit denen dieses Muster realisiert werden kann, denen mühelos noch einige mehr hinzugefügt werden können (cf. den zweiten Block mit Ausdrücken rechts oben in der folgenden Abbildung):

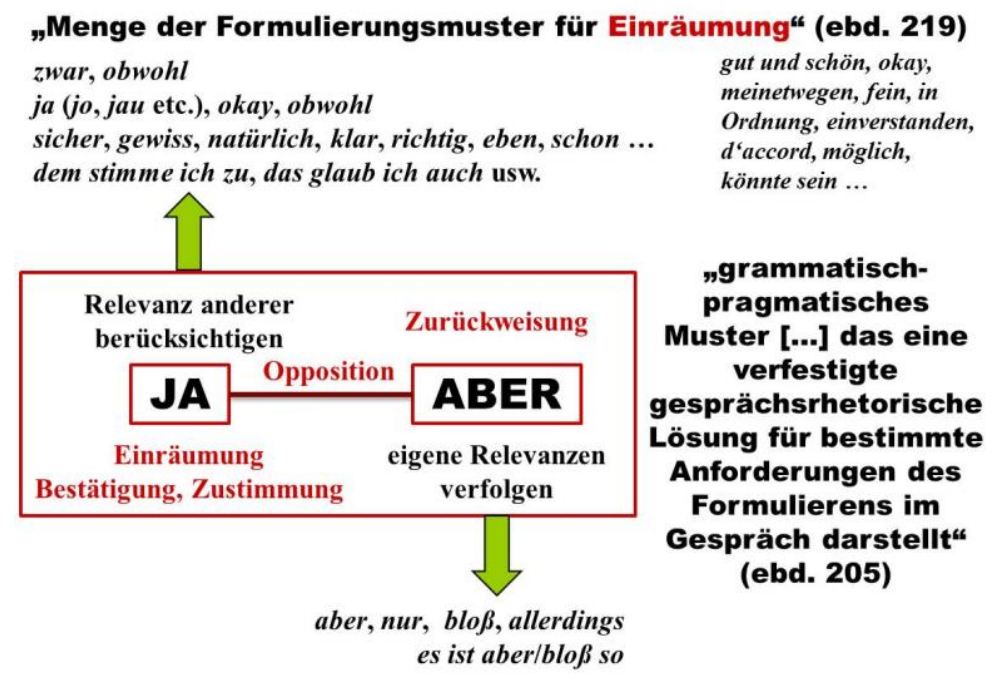

Abbildung 2: Grundlegende Bestimmung der ja-aber-Konstruktion mit Realisierungsbeispielen für beide Teile in Kallmeyer 2007 (Die Beispiele oben rechts habe ich hinzugefügt.)

Warum dieses grammatisch-pragmatische Äußerungsmuster schließlich JA ABER genannt wird, begründet Kallmeyer (nach wie vor) so:

Da ja für den ersten und aber für den zweiten Teil mit Abstand die häufigsten Markierungen sind, wird als allgemeine Kennzeichnung der Funktionen der beiden Teile JA und ABER verwendet, unbeschadet der Tatsache, dass es Funktionsunterschiede in Abhängigkeit von den gewählten Konnektoren und sonstigen Formulierungsmustern gibt.

(Kallmeyer 2007: 206)

\subsection{Schwitalla 2012}

In seiner mittlerweile wohl als Klassiker zu bezeichnenden Einführung in die Beschreibung des gesprochenen Deutsch fasst Schwitalla Ergebnisse der verschiedenen Zugänge zur ja-aberKonstruktion im Kapitel zu syntaktischen Kategorien folgendermaßen zusammen:

\subsection{3 ja und, ja aber und Varianten}

Konjunktionen zu Beginn eines Sprecherbeitrags lassen schon erkennen, wie der Sprecher den Beitrag des vorhergehenden Sprechers einschätzt und was er/sie als Nächstes sagen wird. Bloßes ja schließt zustimmend, zur Kenntnis nehmend an die Vorgängeräußerung an (Strecker 2002, 575): ja aber ist in argumentativen Gesprächen besonders geeignet, um bei thematischer Aufnahme des zuvor Gesagten eine eigene, einschränkende oder widersprechende Einschätzung anzukündigen. Der $j a$-Teil hat bei prosodischer Anbindung an aber nur Gliederungsfunktion für den Beginn des eigenen Beitrags mit thematischem Bezug (vgl. 6.2.2.). Ein akzentuiertes und mög-

und Abstandsdefinitionen), die sich in COSMAS II bisher bewährt haben. Man muss also natürlich wissen, was man sucht, und dafür ist es notwendig, bereits vor der Suche mögliche Realisierungsformen (mindestens eines Teils) der Such-Konstruktion zu kennen. Daran führt, solange nicht streng corpus-driven verfahren wird, m. E. kein Weg vorbei. 
licherweise mit einer Pause abgetrenntes $j a$ macht die Zustimmung stärker (vgl. Barth-Weingarten 2006, 83). Die Konjunktion aber weist auf die andere Meinung voraus. Im Gegensatz zu deutlich widersprechenden Meinungen, die gleich mit einer Negationspartikel eingeleitet werden (nein ich meinte ...; nee mir geht es darum ...), wirkt ja aber gesichtsschonender (Koerfer 1979, 26). Expansionen, Akzentuierungen und intonatorische Gestaltungen von ja geben besondere Einstellungen wieder und stiften ebenfalls für die dann folgenden Äußerungen einen spezifischen Interpretationsrahmen (Willkop 1988, 86-106).

(Schwitalla 2012: 143f.)

Mit der prosodischen Anbindung von ja an aber nennt Schwitalla ein formales Unterscheidungskriterium, das sich funktional auswirken soll: schwächere Anbindung = stärkere Zustimmung, ein Kriterium das auch schon bei Kallmeyer eine Rolle spielte, nämlich ,zusammengezogen artikuliert als ,ja:ber““. (Koerfer 1987: 58). Allerdings analysiert Barth-Weingarten (2006: 83), die Schwitalla hier angibt, bei ihrer Untersuchung von Konzessivität und Adversativität englische Beispiele mit $y E s / y E A h$, well, yes/yeah= und ist daher nur bedingt fürs Deutsche heranzuziehen.

Auch wenn Schwitalla je nach prosodischer Eigenständigkeit dem ja mehr oder weniger zustimmende Bedeutung (im Sinne eines Responsivs) zuweist, bleibt die Analyse von ja aber dennoch kompositional: ja aber als Vorlaufelement zu Beginn des eigenen Turns bei der sequenziellen Organisation von Äußerungen ,,bedeutet thematische Kohärenz zusammen mit Dispräferiertheit“" (Schwitalla 2002: 273).

\subsection{Die Verführungskraft von ja aber}

Die Beschreibungen von Lütten, Koerfer und Kallmeyer haben es möglich gemacht, bestimmte sprachliche Vorkommen analysierend mit dem Namen ja-aber-Konstruktion zu erfassen. Einigen solchen Stellen in konversationsanalytischen Studien soll folgend nachgegangen werden. Im Vordergrund stehen dabei Stellen, an denen die Konstruktion bei der Analyse in ihrer Funktion vorausgesetzt wird, bei denen das Analyseziel - möglicherweise gegen die Meinung der AutorInnen - also nicht darin besteht, herauszufinden, welche Funktion diese Konstruktion in dem ein oder anderen Beispiel hat, sondern die Konstruktion samt Funktionszuschreibung zu identifizieren. ${ }^{9}$ Dass es auch anders geht, zeigt etwa Müller (1997: 320f.) - allerdings wohl eine Ausnahme.

\subsubsection{Spiegel 1995}

In ihrer Dissertation zu Streit-Interaktionen untersucht Spiegel anhand zweier ausführlicher Fallanalysen, welcher Mittel sich zwei Mann-Frau-Paare beim Streiten bedienen. An zwei Stellen taucht ja aber auf: direkt aufeinander folgend in 2.16 im ersten Transkript und in Distanzstellung in $4.08 \mathrm{im}$ zweiten. Zum ersten Vorkommen schreibt sie:

\footnotetext{
9 Übrigens unterliegt man nicht nur in Studien dieser Verführungskraft. So ist in dem Gesprächsanalyse-Lehrbuch von Deppermann etwa zu lesen: ,Jede Beitragskonstruktionseinheit trägt zum Sinn des Gesamtbeitrags bei, bildet aber auch schon selbst eine Teilhandlung. Z. B. stellt der einleitende ,ja'-Teil einer ,ja aber'-Konstruktion ein Zugeständnis, eine Berücksichtigung oder eine Zustimmung dar; er trägt zu einem Widersprechensakt bei, der durch ihn abgeschwächt und höflicher formuliert wird“" (Deppermann $\left.{ }^{4} 2008: 58\right)$ “.
} 
Seine Äußerung Zeile 2.16ff. eröffnet Martin mit ja aber, das einen formalen Bezug an die Vorgängeräußerung bildet und nicht als eine teilweise Zustimmung zu verstehen ist. Der adversative Konjunktor aber signalisiert Widerspruch.

(Spiegel 1995: 77)

Eine solche Analyse basiert darauf, dass man das Vorkommen einer bestimmten sprachlichen Einheit (ja aber) feststellt und ihr sodann eine der beiden Hauptfunktion (nicht-affirmativ adversativ) zuschreibt. Aus dem Transkript selbst geht nämlich nicht hervor, ob hier affirmiert wird oder nicht:

Beispiel (2)

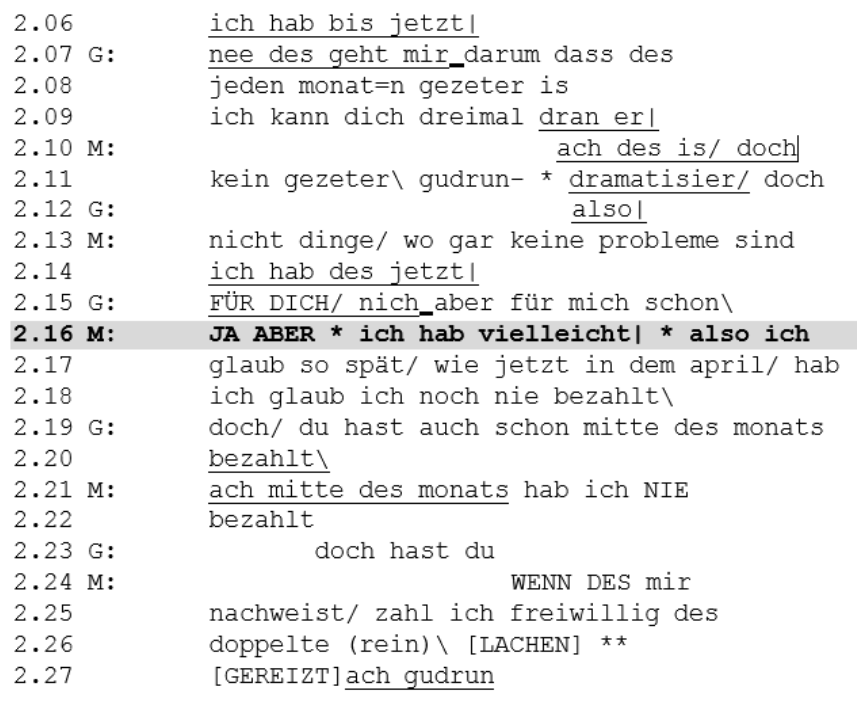

Abbildung 3: Transkriptausschnitt aus Spiegel 1995: 61 (Hervorhebung durch d. Verf.)

Methodisch scheint die Analyse dieser Stelle so rekonstruiert werden zu können: Identifikation des Vorkommens einer ja-aber-Konstruktion, hier in Kontaktstellung, weshalb mit dem ja-Teil nicht affirmiert, sondern gegliedert wird.

Ein ähnliches Vorgehen lässt sich auch bei der Analyse des zweiten Vorkommens der ja-aberKonstruktion erkennen:

Beispiel (3)

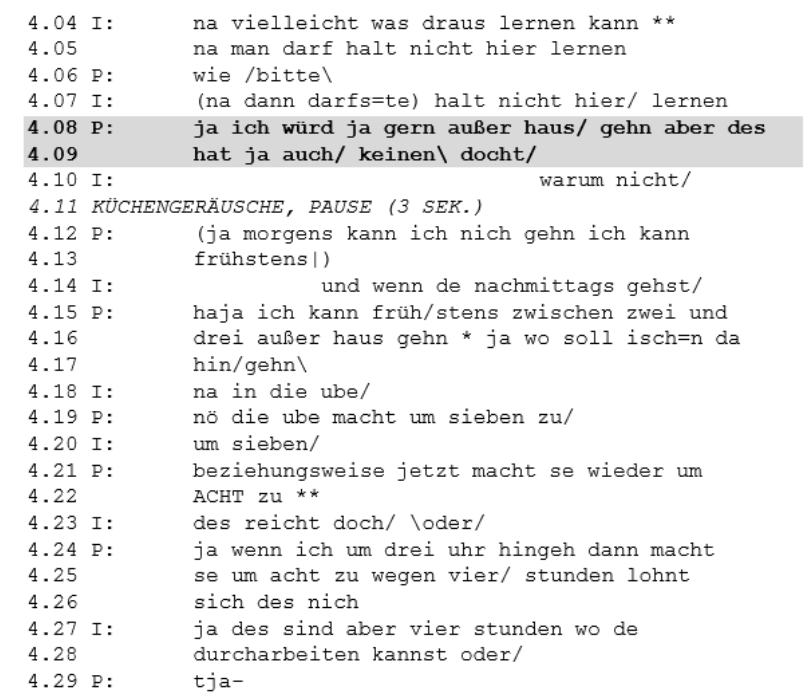


Spiegel schreibt dazu:

Er formuliert seine Aussage mit einer ja-aber-Konstruktion: Die Äußerung wird mit ja eröffnet und konjunktivisch formuliert. Mit dem Vorderglied der ja-aber-Konstruktion versichert Peter Ingrid seine Bereitschaft zur Ausführung ihres Vorschlags und erklärt diesen im nachfolgenden Einräumungsteil für nicht praktikabel. Der Einräumungsteil wird sachlich und ohne Angabe von Personalpronomina formuliert und erhält dadurch und durch die Verwendung der Partikelkombination ja auch, die gemeinsamen Konsens signalisiert, den Status einer Neutralisierung.

(Spiegel 1995: 157)

Ob Peter mit dem ja-Teil seine Bereitschaft versichert, ist dabei alles andere als klar. Schon allein deswegen, weil versichern erst einmal definiert werden müsste: Was ist ,Versichern' als Sprechhandlung? Was tut jemand, der versichert? Nur wenn das geklärt ist, könnte man auch sagen, dass hier versichert würde. Versichern ist ein objektsprachliches Verb, das hier nicht reflektiert als Analyseinstrument verwendet wird. Dabei übernimmt man alle semantisch-polysemen Gebrauchsunschärfen, die objektsprachliche Einheiten mit sich bringen. ${ }^{10}$ Das betrifft auch Vorschlag: Ist „na man darf halt nicht hier lernen“ ein Vorschlag, auf den P mit ,ja ich würd ja gern außer haus/ gehn“",seine Bereitschaft versichernd" reagiert? Jedenfalls scheint wieder das folgende Vorgehen rekonstruiert werden zu können: Identifikation des Vorkommens einer $j a$-aber-Konstruktion, hier in Distanzstellung, weshalb mit dem $j a$-Teil affirmiert wird.

\subsubsection{Gruber 1996}

Dasselbe Problem zeigt sich bei Gruber (1996: 187), dessen Gegenstand ebenfalls Streitgespräche sind.

Beispiel (4)

„JA ABER“

Anders ist die Relation zwischen Bezugs- und Folgeäußerung bei der Verwendung von ,ja aber“. Wie schon Koerfer (1979) darstellt, handelt es sich bei der Verwendung von ,ja aber" auf der interpersonellen Ebene insofern um eine Konfliktvermeidungsstrategie, als damit nicht generell die Bezugsäußerung zurückgewiesen wird.

(Gruber 1996: 187)

\footnotetext{
${ }^{10}$ Versichern müsste nämlich einerseits gegen bekräftigen, beschwören, bestätigen, beteuern, bezeugen usw. (alles Verben, die als Synonyme zu versichern im Duden Bedeutungswörterbuch angeführt sind) abgegrenzt werden und andererseits müsste erklärt werden, warum hier kein Akt von behaupten, mitteilen, informieren usw. vorliegt. Außerdem sollte man auch Einwänden der Art begegnen können, versichern sei eine Sprachhandlung, die vor allem dann zum Zuge kommt, wenn vorher etwas bezweifelt worden ist (cf. etwa Rolf 1997: 159f.). Was antwortet man auf einen solchen Einwand? Im Grunde muss man hier (im Ansatz jedenfalls) sprechakttheoretisch arbeiten (oder sprechakttheoretische Arbeiten konsultieren, etwa Rolf 1983).
} 


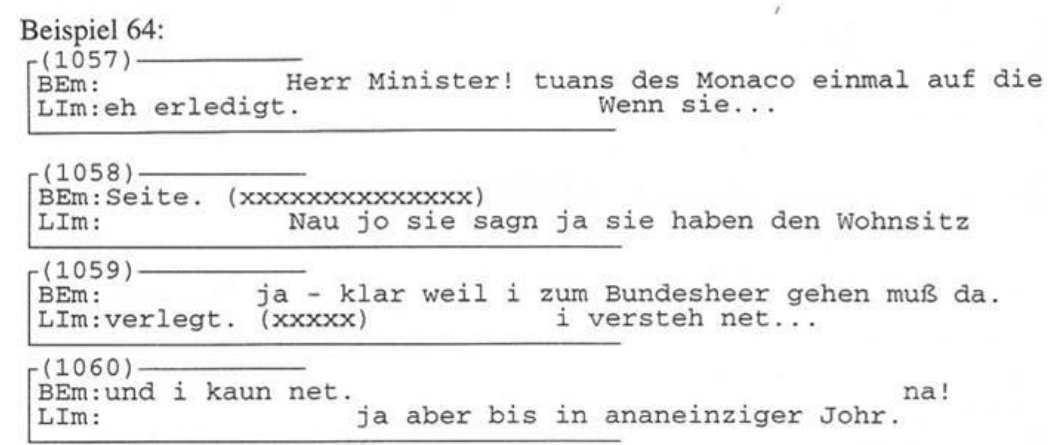

(Club 2, 24.11.88)

Abbildung 5: Gruber 1996: 187f.

Gruber schreibt dazu:

In F1057 widerspricht Berger Lichals Darstellung und fordert ihn auf, dieses Thema einmal wegzulassen. Lichal insistiert jedoch auf seiner Behauptung (F1058/1059), worauf Berger ihm Recht gibt, jedoch als Erklärung seine derzeitige Situation angibt (F1059/1060: ,weil i zum Bundesheer gehen muß da und i kaun net"). Daraufhin widerspricht ihm Lichal in F1060 abermals.

Diesen Widerspruch leitet Lichal mit ,ja aber“ ein. Dadurch ratifiziert er gleichzeitig die Bezugsäußerung und widerspricht ihr in einem Teilaspekt, d.h. er bestätigt, daß Berger in Österreich zum Bundesheer muß, relativiert aber durch die Zeitangabe „,bis ins ananeinziger (,einundneunziger“, H.G.) Jahr“ die „Bedrohlichkeit“ von Bergers Aussage. Im Gegensatz zu „na aber" wird bei der Verwendung von ,ja aber“" zur Einleitung eines Widerspruchs also die Berechtigung der Bezugsäußerung zumindest in einem Teilaspekt anerkannt. „Ja aber“ leitet immer einen Teilwiderspruch ein. Teilweise Anerkennung und Problematisierung der Bezugsäußerung müssen dabei nicht immer auf derselben Ebene liegen [...].

(Gruber 1996:188)

Entgegen jeder methodischen Selbstfestlegung konversationsanalytischen Arbeitens kulminiert Grubers Analyse - in der übrigens auch wieder methodisch nicht reflektiert alltagssprachliche Einheiten wie widersprechen, auffordern, insistieren, Recht geben, Erklärung, bestätigen usw. als Instrument verwendet werden - in dem geradezu apodiktisch anmutenden Urteil, ja aber leite immer einen Teilwiderspruch ein. Das Vorgehen dürfte also wie folgt rekonstruiert werden: Identifikation einer ja-aber-Konstruktion in Kontaktstellung, mit der ein Teilwiderspruch eingeleitet wird, weil mit ja aber immer ein Teilwiderspruch eingeleitet wird.

\subsubsection{Olbertz-Siitonen 2009}

Als letztes Beispiel für die Verführungskraft der wörtlichen Lesart von ja aber sei die Analyse von Olbertz-Siitonen zu dem folgenden Transkriptausschnitt angeführt:

Beispiel (5)

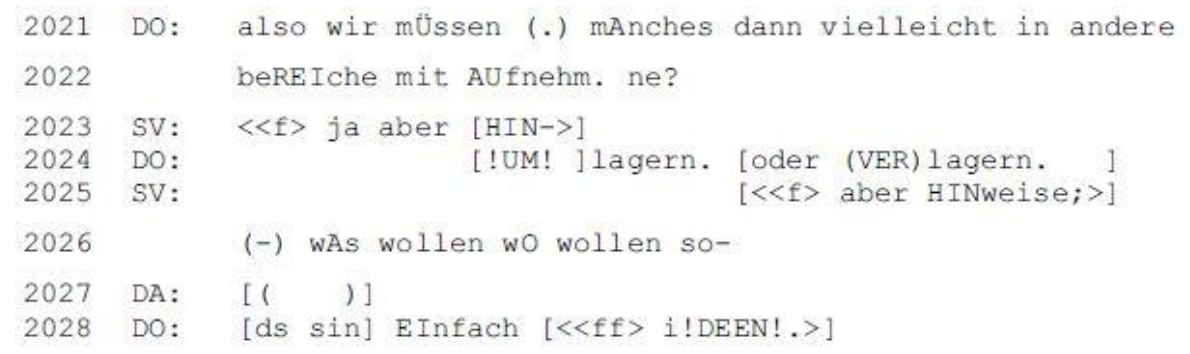

Abbildung 6: Transkriptausschnitt aus Olbertz-Siitonen 2009: 173

ISSN 1615-3014 
Sie schreibt dazu:

Mit seiner ja-aber-Konstruktion markiert er nicht nur Dissens, sondern auch Turnübernahme und Fortsetzungsabsicht (aber kündigt Weiterführung - einen Kontrast - an) ${ }^{112}$ allerdings beginnt Dorothea nach aber wieder zu sprechen, so dass sich die von Sven produzierte Silbe HIN- mit ihrem stark betonten !UM! überlappt (Z. 2023/2024).

(Olbertz-Siitonen 2009: 174)

Dabei ist besonders die Fußnote 112 von Interesse:

Die Funktion von ja aber in Gesprächen wird z. B. ausführlich von Koerfer (1979) behandelt.

(Olbertz-Siitonen 2009: 174)

Hier sind es das bloße Vorkommen von ja aber und das folgende aber HINweise, die als Basis für die Funktionsbestimmung herhalten müssen. Zur Einschätzung, dass aber einen Kontrast ankündigt, kann man jedenfalls aus den Gesprächsdaten - und nur auf diesen Ausschnitt bezieht sich die Analyse - nicht gelangen, es sei denn, man nimmt aber hier wörtlich, ohne Bezug auf den Gesprächsverlauf. Als Indiz für diese Analyseverlegenheit kann schließlich noch die Fußnote herangezogen werden, in der man sich mit Verweis auf die Gewährsperson Koerfer darauf beruft, dass die Funktionen von ja aber erforscht seien. Noch an einer weiteren Stelle in dem Gespräch taucht ja aber auf. Aber auch dort ist das beschriebene Analysevorgehen zu konstatieren, wie an der folgenden Formulierung deutlich wird: „Mit ja aber markiert er Dissens, den er aber nicht ausformuliert.“ (Olbertz-Siitonen 2009: 177). Wenn er den Dissenz nicht ausformuliert, woher weiß man dann, dass er einen Dissens markiert?

\subsection{Meer 2007}

Auch Meer (2007: 18f.) spricht von ja aber als von einem „konventionalisierten Dissensmarker“. Vor dem Hintergrund der Erkenntnisse von Auer/Günthner (2005) geht es in ihrem Aufsatz aber vor allem um die Unterscheidung zweier Vorkommen von $j a$, nämlich als propositional vollwertige Antwortpartikel und damit eigenständige Turnkonstruktionseinheit und als diskursfunktionale Diskurspartikel.

Für die Funktion als Antwortpartikel sprechen dabei die folgenden Kriterien:

- $\quad$ sequenziell sinnvolle Interpretation als Antwort (etwa auf Entscheidungsfragen),

- Realisierung als eigenständige prosodische Einheit,

- $\quad$ potenzielle Erweiterbarkeit um den in der Antwort erfassten Inhalt und

- Weglassprobe, bei der sich keine Änderung des angeschlagenen Tons (etwa aggressiver als mit ja) ergibt.

Für die Funktion als Diskursmarker:

- Integration im Vor-Vorfeld (VVF) einer anderen syntaktischen Konstruktion,

- $\quad$ syntaktische Anbindung/Anbindbarkeit an folgende Einheiten so auch weitere Partikeln wie aber und

- Weglassprobe, bei der die durch ja erzeugte (Ab-)Tönung wegfällt.

Für das Vorkommen von ja als Diskursmarker stellt Meer eine propositional bedeutungsoffene Ambivalenz fest, die darin besteht, „dass ,ja‘ als Element des Vor-Vorfelds keineswegs nur 
positiv bestätigende Funktionen erfüllt. Gerade in konfrontativen Talkshowpassagen leitet die Partikel häufig Vorwürfe oder Widerspruch ein“ (Meer 2007: 2). Wenn ja- und das betrifft dann auch ja aber - im VVF ${ }^{11}$ integriert auftritt, widerspricht dies der (wörtlichen) Lesart, bei der man dem ja ,prinzipiell einen propositional bestätigenden Gehalt zuspricht“ (Meer 2007: 19). Entscheidend für die Einschätzung, ob ja im VVF steht oder nicht, ist wiederum die prosodische Integration. Ist das $j a$ bei einem Vorkommen von $j a$ aber prosodisch eigenständig (und kann es um einen zustimmungsfähigen Inhalt erweitert werden), so handelt es sich um eine Antwortpartikel außerhalb des Satzes und nicht um einen Diskursmarker im VVF des Satzes. Und wenn das so ist, dann wird auch aber nicht als Partikel gebraucht, sondern als Konjunktion:

Zu widersprechen ist aus dieser Perspektive einer Lesart von ,ja aber', die dem ,ja' ohne Beachtung seiner konkreten prosodischen Realisierung prinzipiell einen propositional bestätigenden Gehalt zuspricht. Hierbei ist zu beachten, dass in meinem Korpus durchaus Beispiele zu beobachten sind, in denen einem intonatorisch abgeschlossenen , ja ${ }^{6}$ die Bedeutung einer Bestätigungspartikel (im Sinne von ,ja, Du hast Recht') zukommt. (Beispiel: Mo: ,glaubst du denn so etwas GIBT es, würden das frauen MACHen, ' - Ka: ;JA. aber unter der HAND. '). Dieser Fall ist jedoch deutlich von den zuvor diskutierten zu unterscheiden. So erfüllt das einleitende ,JA. ' in diesem Beispiel auf propositionaler Ebene eindeutig bestätigende Funktionen und ist somit nicht als Diskursmarker, sondern als Antwort- und Bestätigungspartikel zu interpretieren. Zusätzlich ist zu beachten ist, dass auch das anschließende ,aber' kein Diskursmarker ist, sondern in der Funktion einer adversativen Konjunktion gebraucht wird, die auf der Basis der vorhergehenden Bestätigung operiert (,JA. (frauen würden das MACHen,) aber unter der HAND.'). Gerade ein solcher Fall liegt jedoch nicht vor, wenn ,ja aber' intonatorisch integriert im Vor-Vorfeld von Äußerungen auftritt.

(Meer 2007: 19f.)

Während Meer handhab- und belastbare Kriterien für die Unterscheidung verschiedener Verwendungen von ja liefert, scheint sie bei aber allerdings wieder von der Grundfunktion der Konjunktion, Dissens zu markieren, auszugehen. Für ja als Diskursmarker hält sie fest:

Die Partikel erfüllt vorrangig kohäsive Funktionen auf diskurspragmatischer Ebene, indem sie die prinzipielle Bereitschaft zur Interaktion bestätigt und damit deren Nutzung im Sinne einer folgenden Stellungnahme einleitet. Insoweit kommt es verglichen mit der Funktion der Antwort- und Bestätigungspartikel ,ja ${ }^{`}$ zu einer Verlagerung der ursprünglich denotativen Bedeutung der ,Bejahung und Bestätigung` auf eine dominant metapragmatische Ebene.

(Meer 2007: 6)

M. a. W.: Folgt auf den Diskursmarker ein ebenfalls noch im VVF stehendes aber, so zeigt ja mit aber zusammen einen Dissens an, ist ja mit aber geeignet, einen konfrontativen Turn einzuleiten. Ob das aber so ist, ob ja aber also ein konventionalisierter Dissensmarker ist, das müsste selbst noch herausgefunden werden. Die entsprechende Beispielstelle bei Meer lässt eine Entscheidung darüber jedenfalls nicht so ohne Weiteres zu:

\footnotetext{
${ }^{11} \mathrm{Zu}$ weiteren VVF-Besetzungen im gesprochenen Deutsch cf. Auer 1997. Für ja aber, das Auer benutzt, um die Bedeutung/Funktion von im VVF konjunktional (und eben nicht subjunktional) gebrauchtem obwohl oder wobei anzugeben, sieht Auer die Funktion darin, Gegenargumente einzuleiten: „Für die übrigen schriftsprachlichen Subjunktionen, die in der gesprochenen Sprache auch beiordnend gebraucht werden, stehen genauere Untersuchungen noch aus. Obwohl und wobei scheinen oft als Einleitungen für Gegenargumente (etwa im Sinne von andererseits oder ja, aber...) verwendet zu werden.“(Auer 1997: 75f.).
} 
Beispiel (6)

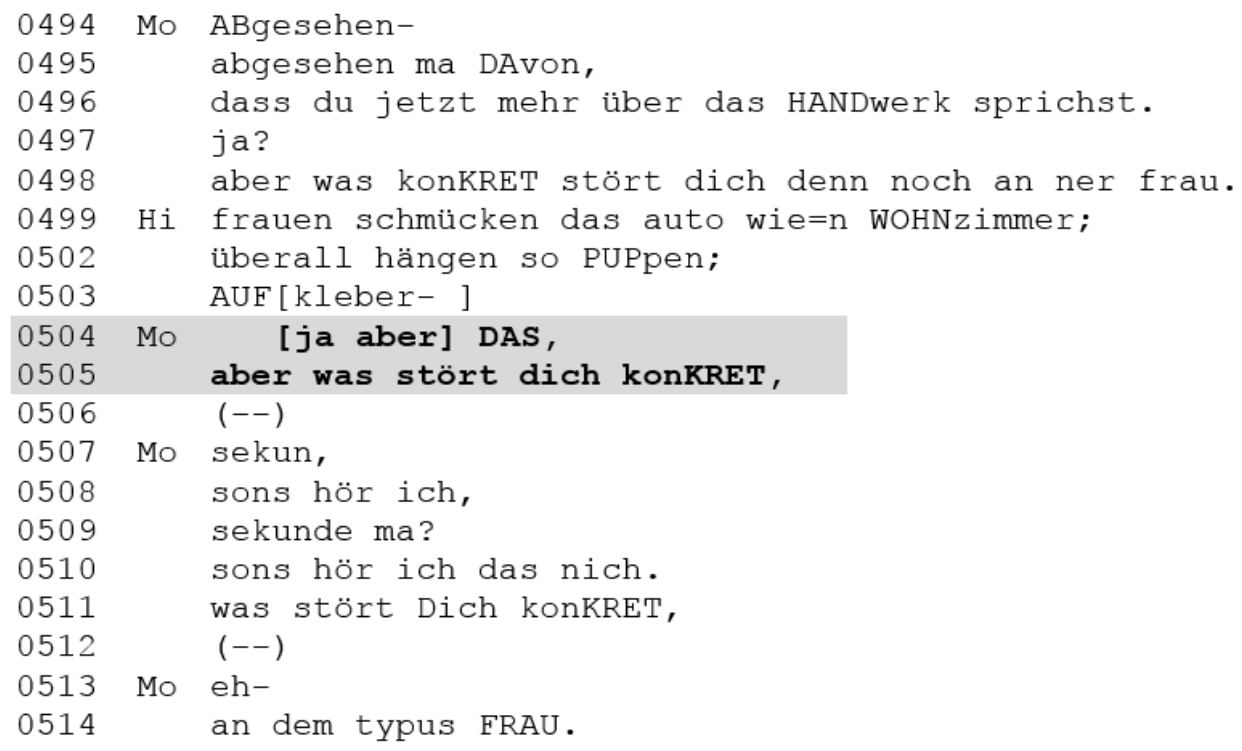

Abbildung 7: Transkriptausschnitt aus Meer 2007: 18 (Hervorhebung durch d. Verf.)

\subsection{Mroczynski 2012}

Mroczynski untersucht Pragmatikalisierungsphänomene anhand der Herausbildung der Diskursmarker wobei, weil und ja. Unter „Pragmatikalisierung“ versteht er dabei einen „graduelle[n] Prozess [...], bei dem ein Ausdruck eine diskursive Funktion übernimmt, dabei allmählich seine ursprüngliche Bedeutung verliert und eine sowohl syntaktische als auch phonetische Eigenständigkeit erfährt“" (Mroczynski 2012: 123). Diskursmarker fasst er folgendermaßen:

Sie erfüllen Aufgaben auf der kommunikativen Ebene, auf der sie multifunktional einsetzbar sind. Sie gliedern Äußerungseinheiten, leiten Korrekturen ein, organisieren den Sprecherwechsel und können dem Beziehungsmanagement dienen. Formal betrachtet, lässt sich sagen, dass Diskursmarker meist durch einzelne Wörter oder kurze nichtflektierte Phrasen realisiert sind.

(ebd.: 123)

Für ja rekonstruiert Mroczynski die Pragmatikalisierung über die folgenden (nicht-linear verstandenen) Stufen:

Wie bisher deutlich geworden ist, weist $j a$ verschiedene synchron erfassbare Verwendungsweisen auf, die als Hinweis auf bestimmte diachrone Wandeltendenzen herangezogen werden können. Diese Wandelschritte können wie folgt dargestellt werden:

1. Antwortpartikel

2. Hörerrückmeldung

3. Inkrementiver Konjunktor

4. Modalpartikel

5. Diskursmarker zur Einleitung von Offensichtlichem

6. Diskursmarker zur Einleitung von Fragen

7. Diskursmarker zur Einleitung von Widersprüchen

(ebd.:185) 
Die Verwendung von ja aber diskutiert Mroczynski schließlich unter Punkt 7. Ja leitet den Widerspruch ein und aber hat verdeutlichende Funktion: „Oft wird der entsprechende Beitrag, der durch $j a$ eingeleitet worden ist, zusätzlich durch ein unmittelbar angehängtes aber verdeutlicht.“(ebd.: 184).

Mroczynski argumentiert für ja aber als kompositional: Die Leistung von ja sei es, einen Widerspruch einzuleiten, die Leistung von aber bestehe darin, diese Funktion zu stärken. Dass ja wörtlich zustimmend ist, als Einleitung von Widersprüchen dann aber nicht mehr zustimmender Natur sein kann, ist für Mroczynski dadurch vereinbar, dass ja im Zuge der Pragmatikalisierung eben auch seinen Gegensinn in die Gesamtbedeutung integriert hat und in konkreten Kontexten aktivieren kann. Insofern sieht Mroczynski hier weiterhin die affirmative Bedeutung von $j a$ am Werk, nur eben gewissermaßen negativiert:

Wie bereits thematisiert, wird in dieser Arbeit die Meinung vertreten, dass selbst bei der Einleitung von Widersprüchen $j a$ seine affirmative Komponente nicht ablegt, weshalb der Behauptung von Meer nicht zugestimmt werden kann. Es ist vielmehr so, dass ja bei dieser Art der Verwendung indirekt seinen ,kontradiktorischen" Sinn erhält. Diese Verwendung ist beispielsweise mit einer ironischen Verwendung des Wortes schnell vergleichbar, das auch im Sinne von, langsam“ verwendet werden kann. Dass eine ironische Verwendung auch einen Sprachwandel auslösen kann, lässt sich an der Entstehung der negativen Gebrauchsweise des Ausdrucks gefälligst ablesen.

(ebd.: 185)

Inwiefern diese ein wenig verwinkelte Argumentation Bestand hat, steht hier nicht zur Debatte. Interessant ist jedenfalls, dass der Versuch unternommen wird, ein kompositionales Verständnis von ja aber auch dann noch zu retten, wenn ja im Grunde nicht ,ja‘, sondern ,nein“ bedeuten soll, indem man beides, ,ja und ,nein', sozusagen als reguläre Polysemie ${ }^{12}$ zur Bedeutung von ja zählt.

\subsection{Zusammenfassung}

\subsubsection{Ja-aber-Konstruktion als funktionales Schema}

Pragmatisch-onomasiologisch geht man von einer Funktionsbestimmung aus und sucht deren Realisierungsformen, die wiederum schließlich auf Unterschiede hin untersucht werden können. Einen solchen Zugang, der auch rhetorisch ${ }^{13}$ genannt werden könnte, wählt Kallmeyer. Für ihn besteht die ja-aber-Konstruktion aus zwei Teilen. Auf einen zustimmenden $j a$-Teil, mit dem an eine vorhergehende Äußerung eines anderen Sprechers angeknüpft werden kann, folgt ein widersprechender aber-Teil, mit dem gegenüber den Inhalten im ja-Teil die eigenen Inhalte in ihrer Relevanz höhergestuft werden. Das, was hier ja-aber-Konstruktion genannt wird, hat einen funktionalen Anker, der strukturell sehr abstrakt bleibt. Ob oder wie diese beiden Teile markiert (z. B. eingeleitet) werden, ob ja und aber selbst auch als Markierung bzw. welche anderen Markierungen insgesamt vorkommen, wie lang die beiden Teile sind, ob die beiden

\footnotetext{
12 Zum Begriff der regulären Polysemie cf. Dobrovol'skij (2006).

13 Cf. Blumenthal (1985), der sich mit der Ja-aber-Taktik als rhetorischer Strategie beschäftigt und zu der folgenden Festlegung kommt: „Ja-aber-Taktiken [...] verfolgen die Absicht, die Darlegung einer eigenen Position aus der Würdigung der Position des Partners heraus zu entwickeln.“ (Blumenthal 1985: 65). Zu den Möglichkeiten, einer Ja-aber-Taktik rhetorisch zu begegnen, cf. u. a. den Podcast von Isabel Garcia auf YouTube (cf. http://www.youtube.com/watch?v=D9jzc6hak-w [13.02.2013]) und Borbonus ( $\left.{ }^{3} 2011\right)$.
} 
Teile überhaupt direkt aufeinander folgen, bleibt dabei unbestimmt. Und muss unbestimmt bleiben, will man nicht von vornherein formale Grenzen eines Bereichs abstecken, der doch möglichst in seiner natürlichen Breite (und nicht in engen strukturellen Grenzen) erforscht werden soll.

\subsubsection{Ja-aber-Konstruktion als Vorkommen von ja aber}

Pragmatisch-semasiologisch geht man von dem Vorkommen bestimmter sprachlicher Einheiten aus und fragt nach ihrer Funktion. Eine solche Perspektivierung haben bspw. Lütten, Koerfer, Meer und Mroczynski (aber natürlich auch Grammatiken wie Zifonun et al. 1997: 373$379^{14}$ oder die Dudengrammatik ${ }^{15}$ ). Für ja und aber stehen hier beispielsweise die Zugehörigkeit zu verschiedenen Wortartklassen (Antwortpartikel, Abtönungspartikel, Diskursmarker, Konjunktion usw.) ebenso zur Debatte wie ihre Funktionen in konkreten Vorkommen. Das, was hier ja-aber-Konstruktion genannt wird, ist eine sprachliche Einheit mit bestimmten strukturellen Ausprägungen, in denen ja und aber vorkommen. Zu klären ist hier, an welcher Position sie vorkommen, ob sie beispielsweise direkt aufeinander folgend oder in Distanzstellung stehen bzw. geäußert werden, ob sie zusammen im VVF oder einzeln je im MF zweier verbundener Sätze/Satzteile angesiedelt sind, wie stark ihre prosodische Eigenständigkeit jeweils ist usw. Das mit der so fokussierten ja-aber-Konstruktion verbundene Erkenntnisinteresse besteht darin herauszufinden, ob es Zusammenhänge zwischen formalen Ausprägungen und bestimmten Funktionen gibt.

In den folgenden Abschnitten werden die Ergebnisse einer korpusbasierten Studie mit letzterer Ausrichtung vorgestellt. Es geht um die Frage von Form-Funktionszusammenhängen sprachlicher Einheiten mit ja aber.

\section{Schriftsprachlicher Zugang zu ja aber}

Obwohl die bisherige Beschäftigung mit ja aber überwiegend aus dem Bereich der gesprochenen Sprache stammt, wird folgend ein Zugang über schriftsprachliches Vorkommen ${ }^{16}$ von ja aber zur Analyse dieser Konstruktion im pragmatisch-semasiologischen Sinn gewählt. Ich gehe dabei grundsätzlich davon aus, dass gesprochene und geschriebene Sprache nicht zwei Systeme ein und derselben Sprache sind, gleichwohl aber natürlich beschreibbare spezifische Charakteristiken aufweisen. Diese Eigenheiten sind m. E. aber grundsätzlich nicht so beschaffen, dass ihnen der mediale Übertritt verwehrt bliebe. M. a. W.: Ich gehe davon aus, dass sich spezifisch

\footnotetext{
${ }^{14}$ Cf. den Absatz zu ja aber im Rahmen der Ausführungen zu ja als Responsiv im turninternen Gebrauch zur Kontinuitätssicherung: ,Relevant für den Diskursaufbau (vor allem in argumentativen Diskussionen) ist die Kombination ja aber, die reaktive Beiträge im Diskurs einleitet und es erlaubt, bei bloß formaler Kohärenzwahrung oder nur eingeschränkter Konvergenz einen die Vorgängeräußerung z. B. problematisierenden, bestreitenden, durch Aspektverschiebung überholenden Beitrag anzuschließen.“(Zifonun et al. 1997: 376).

15 Cf. den $\S 882$ zu den Gesprächspartikeln/Sprechersignalen: „In Startsignalen können Bewertungen oder Kommentierungen des vorangegangenen Redebeitrags enthalten sein: na ja (Skepsis), ja, aber (Einschränkung des zuvor Gesagten), ja, klar (relevanzabschwächend).“ (Dudengrammatik ${ }^{8} 2009:$ 594).

16 In den schriftsprachlichen Belegen befinden sich auch Redewiedergaben. Es ist klar, dass schriftliche Redewiedergaben keine 1:1-Entsprechungen sein können und dass die journalistische oder pressemitteilungsbezogene Verschriftlichung gesprochener Äußerungen zudem einem Redaktionsprozess unterliegt, der in der Regel auf die Produktion schriftsprachlich angemessener Texte ausgerichtet ist. M.a.W.: Die medial schriftlich wiedergegebene Rede unterliegt wohl in der Regel einem Anpassungsprozess in Richtung konzeptuelle Schriftlichkeit mit unterschiedlich weitgehenden redaktionellen Eingriffen.
} 
gesprochensprachliche Konstruktionen im medial Schriftlichen etablieren können und umgekehrt. Ein solches Überschreiten kann zum Beispiel durch Nachahmen spezifischer Merkmale der jeweils anderen konzeptionellen Ebene oder auch durch fortgesetztes Erwähnen und späteren Gebrauch vor sich gehen. Nachvollziehen kann man Übertritte letzteren Typs z. B. bei dem (sicher aber nicht zentralen) Prozess, wie aus geflügelten Worten lexikalische Einheiten werden. Das betrifft bspw. pragmatische Phraseologismen, zu denen etwa auch bald Ich habe fertig zählen könnte (cf. etwa den Artikel „Ich habe fertig. Tasche leer“17 der Verbraucherzentrale Hamburg, in dem nicht mehr angegeben wird, dass hier ein Zitat und ein Wortspiel mit einem weiteren Zitat vorliegt, möglicherweise weil davon ausgegangen wird, dass diese Anspielung noch prominent genug ist, um erkannt zu werden, wobei man aber eben nicht ausschließen kann, dass auch LeserInnen ohne diesen Hintergrund die Überschrift „,verstehen“). Aber auch ohne Bezug zu einzelnen SprecherInnen dürfte die Gebrauchsmembran zwischen dem Schriftlichen und dem Mündlichen für konzeptionelle Spezifika permeabel sein. Eine solche Veränderung (im Sinne eines Übertritts ersteren Typs) kann man derzeit im Schriftlichen möglicherweise bei aus dem eigentlichen Satz ausgeklammerten syntaktischen Zusätzen beobachten. ${ }^{18}$

Eine korpusbasierte Analyse der schriftsprachlichen Verwendung von ja aber hat weiterhin den Vorteil, dass für die Analyse keine Vorannahme der Kompositionalität gemacht werden muss, weil die Belege in aller Regel ausreichend Kotext bieten. Eine solche Vorannahme drängt sich bei gesprochensprachlichen Belegen dagegen zumindest dann auf, wenn mit ja aber z. B. ein Sprecherwechsel versucht wird, der aber scheitert. Man hat dann als Interpretationsrahmen häufig nicht viel mehr als die Äußerung ja aber selbst, was es aber schwer macht, etwas zur Funktion von ja aber über die versuchte Übernahme des Rederechts hinausgehend herauszufinden (cf. etwa oben Beispiel (5)).

Folgend werden also die Ergebnisse einer Studie zu aufeinander folgendem ja aber im Schriftspracharchiv des IDS (über COSMAS II) vorgestellt.

\subsection{Erste quantitative Annäherung}

Für eine Ermittlung der wichtigsten Strukturtypen ist es zunächst einmal wichtig zu erfahren, in welcher syntaktischen Einbettung ja aber vorkommt: im selben Satz, zu Anfang desselben Satzes, am Ende des einen und am Anfang des anderen Satzes usw. Eine solche Erhebung kann man zunächst einmal über zwei Kriterien laufen lassen: Interpunktionszeichen zwischen $j a$ und

\footnotetext{
17 http://www.vzhh.de/ernaehrung/133177/ich-habe-fertig-tasche-leer.aspx. [13.02.2013]

18 Die These, hier handele es sich einfach um ein Übertreten vom Mündlichen ins Schriftliche, oder anders herum: um eine (Re-)Oralisierung des Schriftlichen (cf. Ágel/Hennig 2006: 28 zu aggregativen Satzrandstrukturen), greift dabei wohl zu kurz: „Die vielen als Nachträge zu wertenden Äußerungen, die von ihrem Subordinationsrahmen gelöst werden, oder der intensive Gebrauch des Gedankenstrichs oder Doppelpunkts auch auf der intrasententiellen Ebene sind nicht als einfache Übersetzungen des Mündlichen in das Schriftmedium zu deuten, sondern ahmen stellenweise das für die mündliche Syntax typische ,inkrementelle Anwachsen in der Zeit', die Bevorzugung anderer Spannungsbögen, das beständige Nachtragen, Ergänzen oder Korrigieren, nach." (Schuster 2008: 170; cf. für eine Beispielanalyse solcher syntaktischer Zusätze auch Schwitalla 2012, der Unterschiede und Gemeinsamkeiten gesprochener und geschriebener Zusätze feststellt, selbst aber keine These aufstellt hinsichtlich der Frage, ob hier eine (Re-)Oralität zu konstatieren ist oder nicht; für einen kurzen historischen Überblick cf. Schwitalla 2000). Für ja aber hatte Koerfer (1979) übrigens festgestellt: „Die Erwartung, daß ja aber in monologischen Texten schriftlicher Kommunikation nicht vorkommt, konnte relativ schnell bestätigt werden, so etwa in Zeitungsartikeln und wissenschaftlichen Texten. Wider Erwarten konnte in Texten literarischer Kommunikation, die wie Theaterstücke fiktive Dialoge beinhalten, kein ja aber ermittelt werden.“ (Koerfer 1979: 15).
}

ISSN 1615-3014 
aber (Punkt oder Komma) und Groß- und Kleinschreibung (GKS) von ja bzw. aber. Dabei ergeben sich acht Kombinationsmöglichkeiten, die man über entsprechende Suchketten ${ }^{19}$ einzeln suchen kann. Man erhält folgende Trefferanzahlen (Suche am 08.02.2013):

\begin{tabular}{lccc}
\hline & Punkt & Komma & gesamt \\
\hline ja aber & 73 & $\mathbf{5 9 1 3}$ & 5986 \\
Ja aber & 14 & $\mathbf{6 1 4 6}$ & 6160 \\
ja Aber & $\mathbf{1 4 7 7}$ & 4 & 1481 \\
Ja Aber & $\mathbf{8 2 1}$ & 10 & 831 \\
\hline gesamt & 2385 & 12073 & 14458 \\
\hline
\end{tabular}

Tabelle 1: Trefferanzahl für ja aber in Abhängigkeit von GKS und Interpunktion

Vernachlässigt man einmal die vier Kombinationen, die es zusammen nicht auf $1 \%$ der Gesamttrefferanzahl schaffen (die ohnehin auf besonderen Orthographien, wie der Vollkleinschreibung, beruhen oder Fehler und besondere Kennzeichnungen usw. erfassen), so lassen sich strukturell deutlich zwei Haupt- und zwei Nebentypen ermitteln:

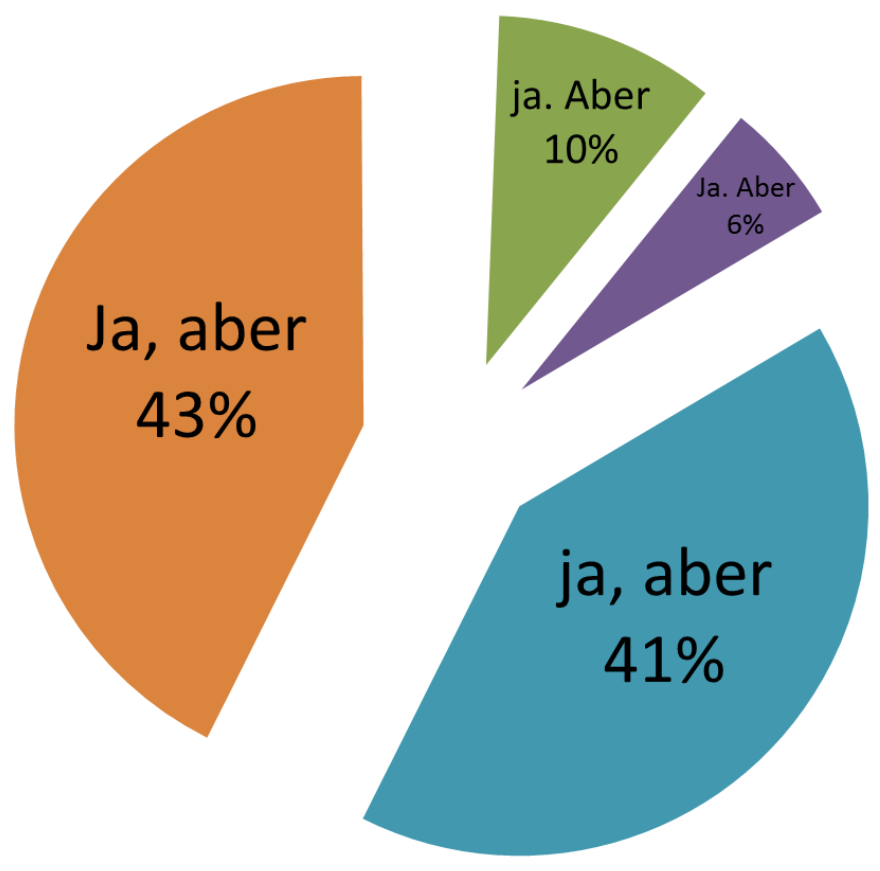

Abbildung 8: Erscheinungsweisen von ja aber hinsichtlich GKS und Interpunktion

Dieses Ergebnis kann man dahingehend interpretieren, dass die Haupterscheinungsweise von direkt aufeinander folgendem ja aber zunächst eine in denselben orthographischen Gesamtsatz eingebettete ist (= Komma zwischen ja und aber, 84\%), wobei die GKS darauf hinweist, dass die satzinitiale Verwendung ( $=J a$, aber) etwa gleich häufig vorkommt wie die kombiniert final-initiale $(=j a$, aber $)$. Darüber hinaus kommt auch noch eine satzübergreifende Verwendung

19 Die Suchketten sind:

\begin{tabular}{lll}
\hline & Punkt & Komma \\
\hline ja aber & ja $/+w 0$. aber & ja $/+w 0$, aber \\
Ja aber & Ja $/+w 0$. aber & Ja $/+w 0$, aber \\
ja Aber & ja $/+w 0$. Aber & ja $/+w 0$, Aber \\
Ja Aber & Ja $/+w 0$. Aber & Ja $/+w 0$, Aber \\
\hline
\end{tabular}


vor (16\%), die sich danach unterteilen lässt, ob ja selbst satzwertig ist (=Ja. Aber) oder nicht (= ja. Aber). Diese Tendenzen wären noch zu präzisieren - Fehlbelege etc. müssten ausgesondert und Substantivierungen extra gezählt werden, bei den finalen Verwendungen müssten noch die Fälle von Komma vor $j a$ gesichtet oder ausgeschlossen werden usw. -, erweisen sich aber bei Stichproben-Überprüfungen in der KWIC-Ansicht als stabil.

An diese quantitative Vorerhebung schließt sich eine qualitative Studie an. Die bisherige Suche hatte die Fälle nicht erfasst, in denen zwischen ja und aber kein Interpunktionszeichen steht. Für die folgende Studie wurde über die einfache Suchkette ,ja aber“ (= gib mir alle Stellen, in denen ja und aber unabhängig von Interpunktionszeichen direkt aufeinander folgen, eingestellte Suchoption: unabhängig von GKS) ein Belegkorpus erstellt. Aus diesem Belegkorpus wurden chronologisch sortiert alle Belege aus dem Jahr 2000 exportiert (Treffer: 10.920, Exportbegrenzung: 10.000) und die ersten 100 (Nichtfehl-)Belege davon zur Einzelanalyse herangezogen. Dieses 100'er Analysekorpus bildet die Grundlage für die qualitative Studie.

Die in der Vorerhebung ermittelten beiden Haupterscheinungsweisen werden ab jetzt unter den folgenden Bezeichnungen geführt:

- $\quad$ TCU (= turn constructional unit) für die satzinitiale Verwendung und

- Gelenk für die kombiniert final-initiale Verwendung.

\subsection{Qualitative Studie}

\subsubsection{Haupterscheinungsweisen in dem 100'er Analysekorpus}

Die beiden Hauptverwendungsweisen zeigen sich auch in dem Analysekorpus, allerdings mit einer anderen quantitativen Gewichtung:

(7) Gelenk $(53 \%)^{20}$

Dafür sei man beim Fremden- und Asylrecht im Wesentlichen seiner Meinung: „Integration ja, aber ein klares Nein zu Neuzuwanderung.“

(8) TCU (34\%)

Stiefel: Dann spielt der Zufall eine grosse Rolle?

Wiedemann: Ja, aber man muss den Zufall auch zulassen.

Zudem konnte die Anzahl der im Analysekorpus vorfindlichen Substantivderivate ermittelt werden. Es scheint so zu sein, dass die Substantivderivate gegenüber der Voruntersuchung zu Lasten der dort als satzinitial eingestuften Verwendungen geht:

(9) Substantivderivat (10\%)

Ein „Ja, aber“ kennzeichnet die Einstellung des Konsumenten zu Bioprodukten.

Folgend werden die Gelenke und die TCU's näher untersucht. Dabei zeigt sich zunächst eine Auffälligkeit in der Verteilung: In über der Hälfte der Fälle (51 zu 35) taucht ja aber als Rede-

\footnotetext{
20 Alle Prozentangaben beziehen sich im Folgenden - solange nichts anderes angegeben ist - immer auf die Grundgesamtheit des 100'er Analysekorpus. Ob dieses Analysekorpus in einem festzulegenden strengen Sinn repräsentativ für das Gesamtvorkommen von ja aber im Schriftspracharchiv ist, kann ohne Anwendung eines statistischen Signifikanztests zwar nicht sicher ermittelt werden. Aber ich gehe davon aus, dass eine chronologische Sortierung der Belege mit anschließender Sichtung der ersten 100 richtigen Positiven der richtige Weg ist, ein zumindest bezogen auf ein ganzes Jahr (hier: das Jahr 2000) repräsentatives Analysekorpus herzustellen. Auf die Anwendung eines Signifikanztests wird aus Gründen, die anderswo (cf. Staffeldt 2012b) niedergelegt sind, verzichtet.
} 
Einheit im Rahmen direkter oder indirekter Redewiedergabe auf, wie auch in den beiden Beispielen (7) und (8) (in der folgenden Tabelle als „Rede“ gekennzeichnet):

\begin{tabular}{lcc}
\hline & Rede & $\neg$ Rede \\
\hline TCU & 29 & $\mathbf{5}$ \\
Gelenk & 22 & 30 \\
\hline
\end{tabular}

Tabelle 2: ja aber in Abhängigkeit von Redewiedergaben

Aussagekräftig ist dabei das geringe Vorkommen als TCU in Belegen ohne Redewiedergabe. Das lässt darauf schließen, dass im Schriftlichen grundlegende Organisationsprinzipien des Gesprochenen (nämlich Dialogizität) jedenfalls nicht so ohne Weiteres adaptiert werden. Andersherum sind die Gelenk-Vorkommen gut verteilt.

Nimmt man nur die beiden häufigsten Fälle, so kann man annäherungsweise sagen: TCU/gesprochen und Gelenk/geschrieben, wobei dann übrig bliebe: Gelenk/auch gesprochen. Grob gesagt, scheint Gelenk damit eher schriftsprachlich zu sein und im Gesprochenen adaptiert zu werden. Solche Fälle wären dann medial gesprochen, konzeptionell aber schriftlich. Betrachtet man ja aber als eine sprachliche Einheit mit vorwiegend pragmatisch zu beschreibender Bedeutung, so erhalten diese Fälle möglicherweise den Charakter von Einheiten des pragmatischen Standards (cf. hierzu die Beiträge in Hagemann/Klein/Staffeldt 2013, vor allem Klein 2013, Deppermann/Helmer 2013 und Staffeldt 2013 sowie Staffeldt 2015).

Bei der weiteren Analyse zeigt sich eine zweite Auffälligkeit. Diese betrifft den Zusammenhang des aber-Teils mit dem vor dem ja-Teil stehenden. Der aber-Teil kann in vielen Fällen nämlich als ausgelagerte Einheit betrachtet werden, die in dem Bezugssatz eine syntaktische Rolle (zum Beispiel als konditionale, kausale, temporale, lokale oder modale Angabe im valenziellen Sinn) einnehmen könnte, wenn sie dort (bspw. nebensatzförmig) stünde. Diese Verwendung bezeichne ich als syntaktisch integrierbar (cf. Abschnitt 3.2.2). Ihr steht eine Verwendung gegenüber, in der der aber-Teil nicht syntaktisch integrierbar ist (cf. Abschnitt 3.2.3).

\subsubsection{Syntaktisch integrierbarer aber-Teil}

Bei syntaktisch integriertem aber-Teil sind zwei Fälle zu unterscheiden, die - wie zu zeigen sein wird - auch funktional verschieden sind. Unterscheidungsmerkmal ist, ob im aber-Teil eine Form der Negation vorkommt (= NEGATION) oder nicht (= EINSCHRÄNKUNG $\left.{ }^{21}\right) . \mathrm{Zu}$ den Negationsmitteln werden hier Negationspartikeln (nein, nicht) ebenso gezählt wie nichtgraduelle negative Indefinita (i. S. v. Dudengrammatik ${ }^{8} 2009$ : 912f., z. B. niemand, kein/er, nichts; cf. hierzu auch Blühdorn 2012: 105-140) und weitere Negationsmöglichkeiten (siehe Beispiel (10)).

(10) Interview, Thema: Bergwerkskatastrophe (Gelenk)

„In meinen kühnsten Träumen hätte ich mir das nicht vorstellen können. Dass das langsam kriecht, ja, aber dass sich darüber neuer Druck aufbaut, das hätte ich ausgeschlossen.“

\footnotetext{
21 Diesen Typ nenne ich „Einschränkung“, weil es so aussieht, als wird im aber-Teil die Gültigkeit des zuvor Gesagten eingeschränkt. Diese Bezeichnung ist ein wenig unglücklich, weil hier - wie wir sehen werden - eher eine komplette Zurückweisung geschieht und eingeschränkt eher in den anderen Fällen wird.
} 
Die syntaktische Integrierbarkeit ist hier allein schon durch die Parallelisierung der beiden dassSätze gegeben, die jeweils als Rechtsherausstellung oder Nachtrag eingestuft werden können, weil sie im Vordersatz pronominal durch das vertreten sind (cf. Schwitalla ${ }^{4} 2012$ : 114f.). Ja ist hier, interpunktorisch durch Komma angezeigt, als Kommentar zu dem ersten dass-Satz als eine Art Responsiv zu betrachten, deren zustimmender Inhalt aus dem Vordersatz kommt: ,dass das langsam kriecht, hätte ich mir vorstellen können'. Aber hingegen markiert: ,dass sich darüber neuer Druck aufbaut, hätte ich ausgeschlossen`. Das Negationselement ergibt sich durch ausgeschlossen. ${ }^{22}$

(11) Filmrezension (Gelenk)

Der arme Regisseur Frank Roddam muss sich da auf Blicke und Rosenbad, auf feuchte Stoffe über Leonor Varelas Körpererhebungen beschränken. Typisch amerikanisch halt. Unmoralisch in allen Belangen bis zum ,geht nicht mehr“", Bruderehe, Ehebruch, Fremdgehen, Freundschaftsverrat ja, aber kein Zentimeterchen mehr Haut, als die Frauenverbände erlauben.

Kompensiert wird diese Doppelmoral mit drastisch ausgemalten Mord- und SchlachtSzenen.

Zentraler Höhepunkt von „Cleopatra I“ ist wohl die breit dargestellte Hockgeburt von Caesars einzigem männlichen Spross, den der römische Senat einen Bastard, Brutus dessen Mutter eine Hure schimpft.

Auch hier haben wir es wieder mit einem finalen ja zu tun. Die im aber-Teil vorkommende Negation ist kein. Dass der aber-Teil syntaktisch integrierbar ist, kann durch einen Weglasstest gezeigt werden: Unmoralisch in allen Belangen bis zum ,,geht nicht mehr “, aber kein Zentimeterchen mehr Haut, als die Frauenverbände erlauben. Ohne syntaktischen Verlust kann der jaTeil weggelassen werden, der aber-Teil ist also jedenfalls integriert. Dieser Test funktioniert übrigens auch in (10).

(12) Feature über Spürhunde (TCU)

Im dritten wird das Training auf ein großes Schneefeld mit vielen Hunden zu suchenden Menschen und Gegenständen ausgedehnt.

Harte Arbeit für die Spürnasen? Ja, aber der Hund darf dabei nie die angeborene Freude am Suchen und Finden verlieren, nie darf ihn sein Herr überfordern. Das ist besonders schwer, wenn es um den Transport des Tieres zum Unglücksort geht. Wie beispielsweise transportiert man einen ausgewachsenen Schäferhund in einem Schlepplift?

Hier wird eine Frage-Antwort-Sequenz inszeniert. Die Antwort wird mit ja aber eingeleitet, in dem aber-Teil ist das zweimalige nie das Negationselement.

\footnotetext{
22 Neben den Negationspartikeln und den negativen Indefinita sind - so etwa in der IDS-Grammatik - auch andere Ausdrücke im Bereich der Negation anzusiedeln, so z. B. „Verben und Redewendungen, denen eine der Negation entsprechende Wirkung zuerkannt wird“ (Zifonun et al. 1997: 848). So würde leugnen eine negative Wirkung haben, weil leugnen 'nicht zugeben' bedeuten würde. Dasselbe muss dann aber auch für die das Prädikativum ausgeschlossen sein gelten, das nämlich als ,nicht eingeschlossen sein'verstanden werden kann. Sein Negationspotential entfaltet ausgeschlossen sein hier in Bezug auf sich vorstellen können, das hier in Verbindung mit ausgeschlossen wohl als ,hätte ich mir nicht vorstellen können‘ o. ä. zu verstehen ist (wie ja auch im Vorsatz formuliert ist).
} 
Auch wenn die Gelenk-Vorkommen sicher nicht die in der Literatur beschriebenen ja-aberVorkommen sind, so kann doch in diesen Gelenk- oder TCU-Fällen mit syntaktisch integrierbarem aber-Teil, in dem eine Negation enthalten ist, noch am ehesten die klassische Funktionsbeschreibung gesehen werden: Der ja-Teil stimmt zu (bejaht), der aber-Teil widerspricht (verneint). Und es scheint auch zu gelten, dass der aber-Teil in seiner Relevanz höher einzuschätzen ist als der ja-Teil. Die ja-aber-Konstruktion leistet hier ein Splitting in Bezug auf Vorhergehendes. Das Vorhergehende wird nicht gänzlich verneint, sondern nur ein Teil davon. Die jaaber-Konstruktion schafft allerdings erst die Möglichkeit des Teilwiderspruchs, indem sie die vorherige Ganzheit aufteilt. Deutlich wird das in den Fällen des - wie man es nennen könnte offenen Splittings wie im Beispiel (7), das hier als (13) wiederholt wird:

(13) Bericht (Politik)

Dafür sei man beim Fremden- und Asylrecht im Wesentlichen seiner Meinung: „Integration ja, aber ein klares Nein zu Neuzuwanderung. “

Das Oberthema „Fremden- und Asylrecht“ wird in zwei Unterthemen aufgeteilt, nämlich „Integration“ und „Neuzuwanderung“. Es ist alles andere als klar, dass das Oberthema so gesplittet ist oder gesplittet werden muss. Das leistet erst die ja-aber-Konstruktion. Dadurch wird es möglich, innerhalb eines Bereiches sich für den einen und gegen den anderen Teil dieses Bereichs zu entscheiden. Man könnte den Zusammenhang folgendermaßen veranschaulichen (daneben gleich die entsprechende Analyse für Beispiel (10)):

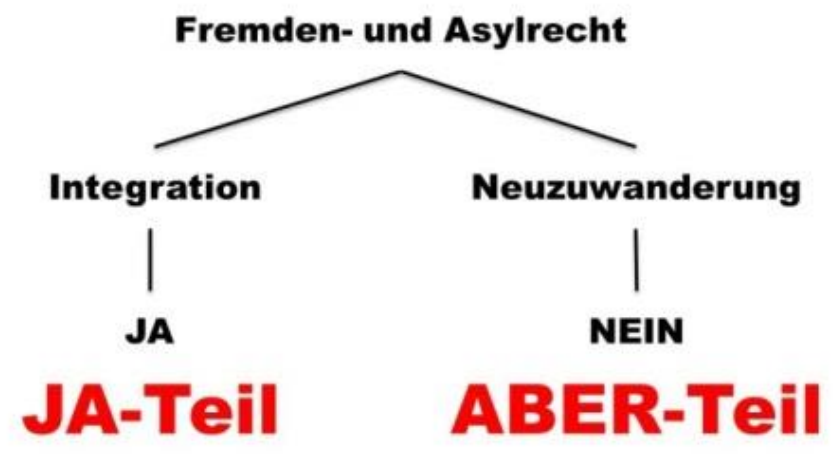

Abbildung 9: Offenes Splitting (Beispiel (13))

sich vorstellen können

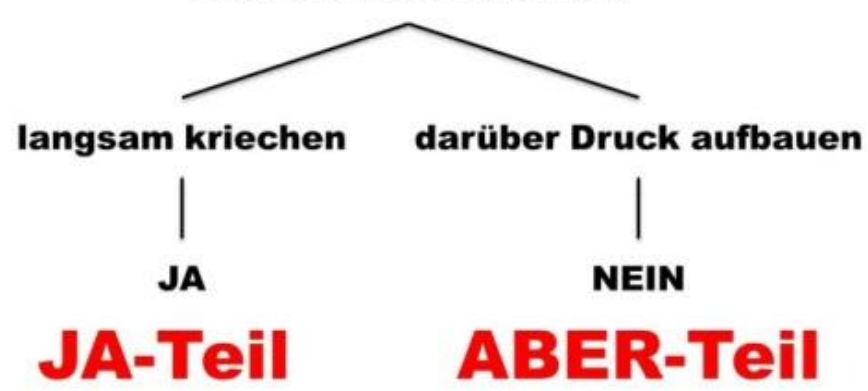

Abbildung 10: Offenes Splitting (Beispiel (10)) 
Neben einem solchermaßen offenen Splitting gibt es aber auch Fälle eines halb verdeckten Splittings. Und hier sehe ich die Möglichkeit, eine eigenständige Funktion von aber zu postulieren. Während nämlich in den Fällen des offenen Splittings die Adversativität im Grunde durch die Negation hergestellt wird, $j a$ als Responsiv zustimmender Natur ist und ja aber insgesamt als Splitter fungiert, so hat - geht man einmal von dem Schema in den Abbildungen 15 und 16 als Analyse-Portfolio aus - aber in den Fällen (halb oder voll) verdeckten Splittings eine gut beschreibbare Aufgabe. Nehmen wir hierfür das folgende Beispiel (14):

(14) Interview eines Kandidaten für eine Wahl

„Ich finde es wichtig, dass die junge Generation Verantwortung übernimmt. Aber ich bin dagegen, dass nur eine Generation bevorzugt wird. Solidarität unter verschiedenen Altersgruppen ja, aber nicht einseitige Massnahmen zugunsten einzelner Bevölkerungsschichten."

Hier liegen, nimmt man Kallmeyers Perspektive ein, zwei nacheinander geschaltete ja-aberKonstruktionen vor. Durch diese Parallelisierung der ersten beiden Sätze mit der ja-aber-Konstruktion im dritten wird es möglich, jeweils einen Zusammenhang herzustellen zwischen dem ersten Satz und dem ja-Teil im dritten sowie dem zweiten Satz, der ja auch durch aber eingeleitet wird, und dem aber-Teil im dritten. Wenn man nun verstehen will, wofür der Kandidat ist, wozu der Kandidat $j a$ sagt, denn zum ganzen Oberthema sagt er ja gerade nicht $j a$, muss man das Gegenteil zu dem im aber-Teil negierten Inhalt herstellen, was bedeutet: die Negation sozusagen rückgängig machen. Man könnte auch sagen, dass man als LeserIn ein Gegenteil zu ,einseitige Maßnahmen zugunsten einzelner Bevölkerungsschichten' (im Schaubild kurz als „einseitige Bevorzugung“) finden muss, was übrigens gar nicht so einfach ist und möglicherweise eher vage mit, ausgewogene Belastung' (statt ',zweiseitige Bevorzugung') o. ä. angegeben werden müsste:

\section{Solidarität unter verschiedenen Altersgruppen}

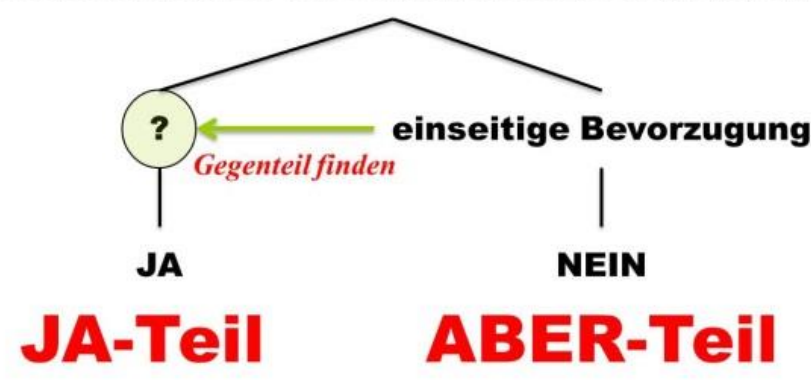

Abbildung 11: Halb verdecktes Splitting (Beispiel (14))

Das Splitting kann übrigens auch voll verdeckt sein. Von der Struktur der ja-aber-Konstruktion bleibt dann nur das Grundgerüst übrig. Etwa wie in Beispiel (15):

(15) Zwischenüberschrift

Ja, aber nicht so

Wenn die obigen Beschreibungen richtig sind, und die Grundfunktion der ja-aber-Konstruktion bei integrierbarem aber-Teil das Splitting eines Oberthemas ist, wodurch ein teilweises Ja-Sagen und ein teilweises Nein-Sagen möglich wird (kurz: Pro-kontra-Splitting oder Dafür-dagegen-Splitting), läuft die Funktion dieser Zwischenüberschrift auf eine dreifache Suchanweisung hinaus: 


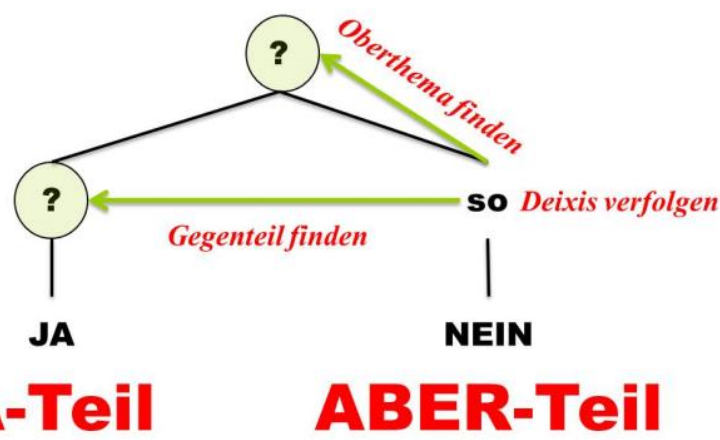

\section{Abbildung 12: Voll verdecktes Splitting (Beispiel (15))}

Die nicht-negierten Fälle funktionieren spiegelbildlich:

(16) Bericht über Reaktionen auf die Ankündigung eines Schulstreiks „Demonstrieren ja, aber in der Freizeit“"

Der Schul-Streik gegen die neue Regierung, den eine Schülerinitiative für Freitag angekündigt hat, stößt auf harsche Kritik.

Der aber-Teil ist syntaktisch integriert (selbst ohne ja aber: Demonstrieren in der Freizeit), im aber-Teil liegt jedoch keine Negation vor. Wir haben es hier wieder mit einem Splitting zu tun. Denn um zu verstehen, was hier gemeint ist, muss ein Gegenteil zu dem Inhalt im aber-Teil gefunden werden, also ein Gegenteil zu in der Freizeit:

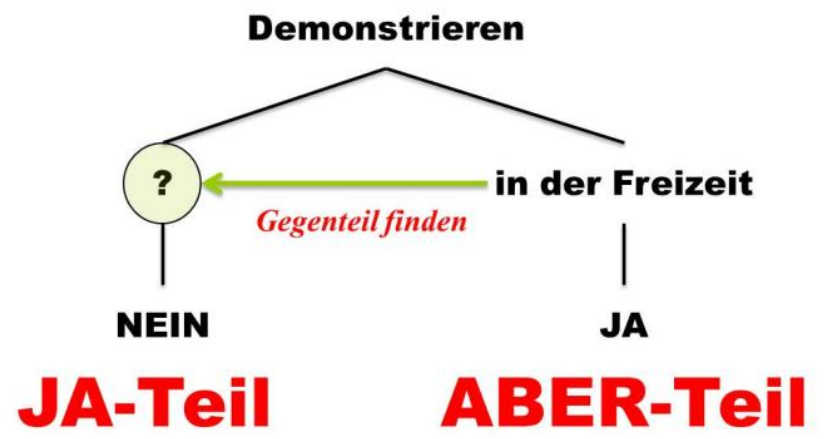

Abbildung 13: Halb verdecktes Splitting (Beispiel (16))

Das einfachste Gegenteil erreicht man natürlich durch Verneinung nicht in der Freizeit, was vorausgesetzt es gibt nur Freizeit und Schulzeit, wobei tertium non datur gilt - bedeutet: ,in der Schulzeit'. Wenn das Dafür-dagegen-Splitting auch hier gilt, heißt das: zu demonstrieren in der Freizeit sagt man ja (dafür), zu demonstrieren in der Schulzeit sagt man nein (dagegen). Das ist argumentativ aber natürlich eine Finte, denn in der Freizeit streiken ist eine contradictio in adjecto, weshalb wohl auch streiken zu demonstrieren umgedeutet wurde.

Genau besehen sagt man in einem solchen Fall also mit dem ja-Teil ,nein'. Mir scheint, dass hier keinerlei Zustimmung zu der fremden Position mehr auszumachen ist. Wer eine solche Konstruktion wählt, tut dies, um ,nein` zu sagen. Oder um es mit Langacker (1987: 183-189) zu sagen: die Seite demonstrieren nein ist profiliert.

Man könnte, und das hat dieser Gruppe ihren Namen gegeben, die Fälle auch als Einschränkung einer Proposition auffassen, wie an den folgenden Belegen gezeigt wird: 
(18) Kausale Einschränkung Kannst du vom Snowboarden leben?

Martin: Ja, aber nur weil ich bis vor wenigen Monaten gearbeitet habe und noch Geld auf der Seite habe.

(19) Temporale Einschränkung

Sparen ja, aber...

Sparen ist schon recht, Herr Minister, aber alles zur rechten Zeit, und das haben Sie die ganze Zeit mit Ihrer Koalitionsregierung nicht verstanden. [Bezug: temporale Angabe zu V]

(20) Lokale Einschränkung

Louis: „Die Personenwagen lassen wir durch und auch die Lastwagen, die aus Frankreich ausreisen. Nur die einreisenden Camions nicht", erklärte Daniel Portmann, einer der französischen Blockierer vor Ort. Europa ja, aber europaweit „La France s'isole isolons la France!, heisst unser Slogan“, meinte er. Wenn die Regierung, vor allem Arbeitsministerin Aubry, mit ihrem 35-Stunden-Woche-Gesetz und der ständigen Verteuerung des Diesel-Treibstoffs dem französischen Transportgewerbe die Geschäfte verderbe, müssten sich die Camion-Unternehmer eben wehren, so Patron Portmann, der in Mülhausen eine Flotte von 100 LKWs betreibt.

(21) Modale Einschränkung

Wer nicht kommuniziert, wird vergessen - von der „financial community“ (Analysten, Wirtschafts-Journalisten oder Prognostikern usw...) - und von den Kunden. Das ist längst bekannt und erforscht. ComTelco hat einen anderen Ansatz: Kommunizieren ja, aber effizient. In den USA haben immer mehr Unternehmen begonnen, die Telefongespräche der Mitarbeiterinnen und Mitarbeiter zu röntgen. Die Zahl der Anrufe, die persönlichen Interessen und jenen der Firma dienen, klaffen erstaunlich oft auseinander.

Was die nein-Profilierung angeht, so müsste dann also stimmen:

- In (18) sagt man, dass man nicht allein vom Snowboarden leben konnte,

- $\quad$ in (19) sagt man, dass man jetzt nicht hätte sparen sollen,

- $\quad$ in (20) sagt man, dass Europa nicht funktioniert, wenn nicht alle (also auch Frankreich) mitmachen, und

- $\quad$ in (21) sagt man, dass man nicht ineffizient (= zu viel privat) im Rahmen von Mitarbeiter-Telefongesprächen kommunizieren soll.

Zusammenfassen lässt sich die Aufteilung beim Dafür-dagegen-Splitting in den Fällen von syntaktischer Integrierbarkeit des aber-Teils mit der folgenden Abbildung: 


\section{Funktion}

\section{Dafür-dagegen-Splitting}

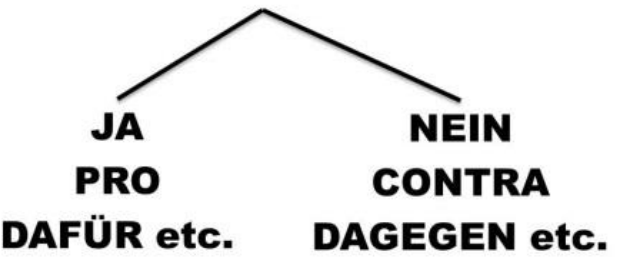

bei Negation im ABER-Teil:

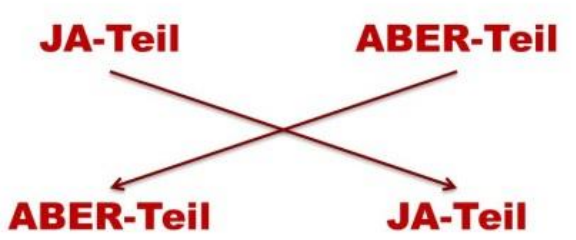

Abbildung 14: Das Dafür-dagegen-Splitting bei ja aber mit syntaktisch integrierbarem aber-Teil

Von der Verteilung her sind es stärker die Gelenk-Fälle, bei denen ein solcher Form-Funktionszusammenhang vorliegt, und mehr NEGATIONsfälle. Deutlich dispräferiert sind die Fälle mit TCU ohne Negation im aber-Teil:

\begin{tabular}{lcc}
\hline & TCU & Gelenk \\
\hline NEGATION & 10 & 23 \\
EINSCHRÄNKUNG & 3 & 14 \\
\hline
\end{tabular}

Tabelle 3: Verteilung der beiden Vorkommensvarianten von ja aber mit syntaktisch integrierbarem aberTeil auf die beiden Haupterscheinungsweisen

Wenn die Beschreibung richtig ist, dann eignet sich diese ja-aber-Konstruktion mit ihrem Dafür-dagegen-Splitting bestens dazu, eine (abschließende) Stellungnahme zu etwas abzugeben. Hier sehe ich auch das Haupteinsatzgebiet für eine solche Konstruktion. Es geht nicht vorrangig darum, zu widersprechen oder die Relevanz des selbst Gesagten hochzustufen, sondern darum, zu einem bestimmten Thema Stellung zu beziehen, d. h.: klar zu machen, wogegen und wofür man bei diesem Thema ist. Sei das Thema textuell gegeben oder sei es selbst gesetzt und monologisch prozessiert oder sei es in der Vorgängeräußerung zu finden und damit dialogisch prozessiert.

\subsubsection{Syntaktisch nicht integrierbarer aber-Teil}

Während bei den Fällen mit syntaktisch integrierbarem aber-Teil ein gesetztes Thema gesplittet wird, kann man mit einer ja-aber-Konstruktion mit syntaktisch nicht integrierbarem aber-Teil Propositionen hinzufügen. Da Illokutionen über Propositionen laufen, sind diese Konstruktionen funktional nicht auf den Bereich der Meinungskundgabe dafür/dagegen festgelegt. Mit ihnen können vielmehr unterschiedliche Sprechhandlungen vollzogen werden. Hierfür seien in der Folge nur ein paar Beispiele genannt, deren genaue Funktionsbestimmung noch aussteht (und auch ein im Grunde eigenständiges Thema wäre). Die Bezeichnungen für die Funktionen sind daher als vorläufig zu verstehen. 
(22) annehmen, Ausdruck einer Vermutung

Bleibt es beim Sitz der Kunstakademie in Andernach?

Schäfer-Schällhammer: Ich denke ja. Aber ich kann mir auch vorstellen, dass in Mayen und/oder Mendig Räume für die Steinbildhauerei von der Kunstakademie eingerichtet werden. Grafik und Druckgrafik könnten in Lahnstein platziert werden.

(23) rechtfertigen

Viele junge Spieler sind zu ungeduldig und nicht bereit, über einen längeren Zeitraum alles zu geben. Konstanz hat, bei allen positiven Anlässen, zuletzt auch der Borussia gefehlt.

Ja, aber wir mussten auch erst einmal den freien Fall stoppen.

(24) ein Gegenargument anführen

Buschor: Kommen Sie einmal mit mir in die Regierungsratssitzung, dann wissen Sie, warum da so viele Zahlen drin stehen.

Vollenweider: Ja, aber mit diesem Schriftstück verkauft sich die Reform schlecht.

(25) Perspektivierung 23

Als ihm der Richter vorhielt, dass es am Ende immerhin 18 Prozent der Gesamtfördermenge waren, hatte der Ex-Betriebsleiter geantwortet: „Ja, aber es ist immer weniger geworden. " Schwarzabbau war in Lassing nichts Neues.

Bezogen auf die strukturellen Haupterscheinungsweisen sind mehr TCU's als Gelenke mit dieser Form-Funktionsausprägung anzutreffen. In der Gesamtübersicht:

\begin{tabular}{lcc}
\hline & TCU & Gelenk \\
\hline NEGATION & 10 & 23 \\
EINSCHRÄNKUNG & 3 & 14 \\
HINZUFÜGUNG & 18 & 10 \\
\hline Gesamt & 31 & 47 \\
\hline
\end{tabular}

Tabelle 4: Verteilung der Vorkommensvarianten von ja aber auf die beiden Haupterscheinungsweisen

\subsubsection{Zusammenfassung}

Für die hier untersuchte ja-aber-Konstruktion in Kontaktstellung, bei der ja und aber unmittelbar aufeinander folgen, aber durch Interpunktionszeichen voneinander getrennt sein können, lassen sich zwei Hauptverwendungen ausmachen: das Splitting und die Hinzufügung. Bei dem Splitting ist der aber-Teil syntaktisch integrierbar, bei der Hinzufügung nicht. Die eigentliche Leistung des Splittings besteht darin, ein Oberthema aufzuteilen, sodass Teilthemen verhandelbar werden. Je nachdem, ob der syntaktisch integrierbare aber-Teil eine Negation aufweist oder nicht, kann das Splitting in die Fälle von NEGATION (mit Negation) und EINSCHRÄNKUNG (ohne Negation) unterteilt werden. Die Negationsfälle sind dabei gut für Meinungskundgaben

\footnotetext{
${ }^{23}$ Hier liegt eine ähnliche Funktion vor, wie sie auch für (oder) besser gesagt (cf. Staffeldt 2012a) festgestellt werden kann: Es geht darum, einen Sachverhalt aus einer bestimmten Perspektive zu sehen. Im Falle von (25) ist die Perspektive des Richters punktuell (,es waren 18\% Schwarzabbau'), die des Ex-Betriebsleiters dagegen linear (,es waren vorher mehr und wir haben es geschafft, den Schwarzabbau auf 18\% zu senken'), wodurch der Sachverhalt (18\% Schwarzabbau) in einem besseren Licht dasteht.
} 
oder Stellungnahmen zu einem bestimmten Thema geeignet. Bei den einschränkenden Fällen geht es eher darum, nein zu einem bestimmten Teilthema zu sagen, wobei die Setzung des anderen Teilthemas, der Einschränkung, u. U. eine rhetorische Finte ist. In der Übersicht:

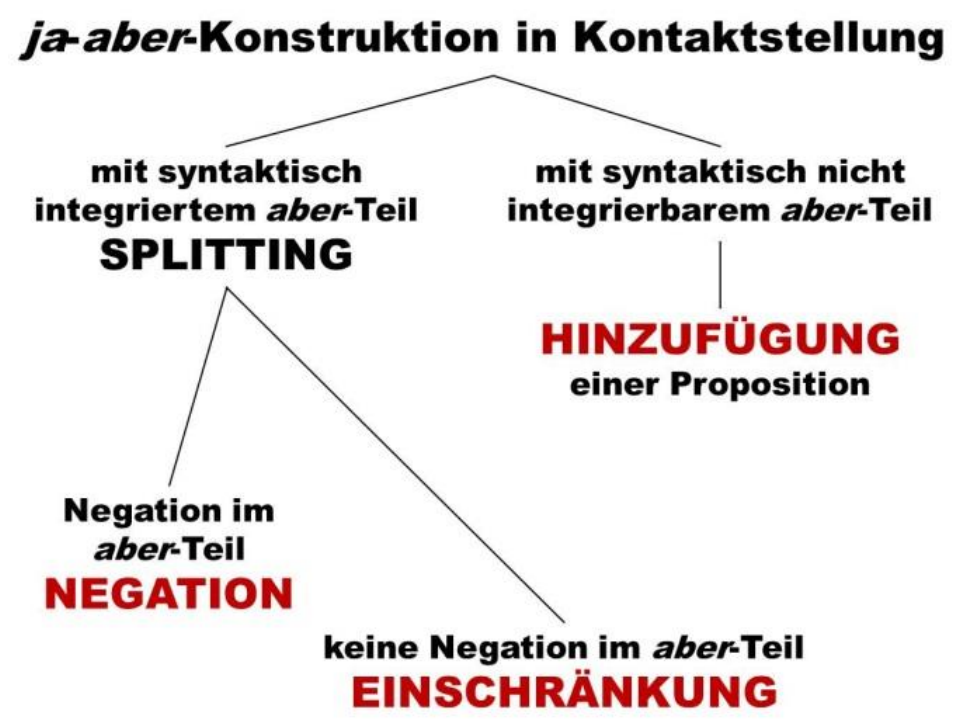

Abbildung 15: Untertypen der ja-aber-Konstruktion in der Übersicht

\section{Drei gesprochensprachliche Beispiele}

Anhand dreier Beispiele wird folgend kurz demonstriert, dass sich die Ergebnisse des schriftsprachlichen Zugangs auch auf gesprochensprachliche Vorkommen der so bestimmten ja-aberKonstruktion anwenden lassen.

\subsection{Telefongespräch}

Das erste Beispiel stammt aus Brons-Albert (1984: 151f.). Es handelt sich um ein Telefongespräch. C, eine 24 Jahre alte Studentin, ruft bei A, einer 25 Jahre alten wissenschaftlichen Hilfskraft und B, einem 29 Jahre alten Grafiker an. A und B sind miteinander verheiratet, C und B miteinander befreundet. $C$ spricht zunächst mit A und teilt mit, dass die Schreibmaschine schon bei $C$ ist. Den größten Teil des Gesprächs bestreiten dann aber $C$ und $B$, wobei mehrere verschiedene Themen abgehandelt werden. Das letzte große Thema vor dem hier interessierenden war die Diät von B und schließlich die Nachfrage von C, ob A und B nicht mal zu Besuch zu C kommen wollen, es könne dann auch Käsefondue geben. B antwortet, sie kämen, wenn B wieder was essen darf. Schließlich gleitet das Thema aus mit einem eher allgemein gehaltenen Vergleich von Käse- und Fleischfondue.

Für einen schnelleren Zugriff habe ich die entscheidenden Stellen in dem folgenden Transkriptausschnitt bereits markiert: 
Beispiel (26)



Abbildung 16: Mit Markierungen und Zeilenzählung aufbereiteter Ausschnitt aus einem Transkript eines Telefongesprächs (aus Brons-Albert 1984: 151f.)

In 4 beginnt ein neues Thema in diesem Gespräch, das bis 24 andauert (rote Umrahmung). Danach folgt wieder ein neues Thema. In beiden Fällen wird die Setzung des neuen Themas durch ein initiales aber eingeleitet. Innerhalb des gesetzten Themas ist an fünf Stellen $(8,11$, 16, 19 und 21) jeweils ein initiales ja anzutreffen. Während initiales aber hier also Themensetzungsfunktion zu haben scheint, so scheint initiales $j a$ innerhalb eines Themas eine fortführende Funktion zu haben.

Eine ja-aber-Konstruktion im Sinne Kallmeyers ist an drei Stellen (hier durch Pfeile markiert) anzutreffen. Darunter befindet sich auch eine in Kontaktstellung (21). Ja aber taucht hier in Gestalt einer TCU auf, wobei der aber-Teil syntaktisch nicht integrierbar ist. Es handelt sich also um einen Fall von HINZUFÜGUNG. Ein solcher ist illokutionär offen. Da in dem zweiten Satz von 21 eine konvers identische Proposition zu 4 zu konstatieren ist, dürften diese beiden Teile zusammenhängen. Die These lautet: Was immer 4 ist, in 21 wird ein Gegenakt vollzogen. Ist 4 eine Art Beschimpfung, ist 21 insgesamt eine Gegenbeschimpfung. Ist 4 eine Art Vorwurf, ist 21 insgesamt ein Gegenvorwurf. Ist 4 eine Art Schuldzuweisung, ist 21 eine Gegenschuldzuweisung. Unterstützt wird diese Interpretation durch die nachfolgenden Turns, in denen in einem scherzhaften Interaktionsmodus (24: (lacht)) die Schuldfrage geklärt wird. 
Der mit der ja-aber-Konstruktion eingeleitete Satz scheint ebenfalls in dem Dienste der Konverse zu stehen. Mit Rolf (1997: 226) könnte hier ,eine Entschuldigung vorbringen' angenommen werden: Man nennt einen Umstand, aus dem klar wird, dass man für den fraglichen Umstand nicht verantwortlich gemacht werden kann, man also nicht schuld ist. Im Gegenteil: Damit wird die Schuldfrage nämlich direkt an den ursprünglichen Schuldzuweiser zurückgewiesen.

\subsection{Mindestlohn}

Bei dem folgenden Beispiel handelt es sich um eine Antwort im Rahmen einer Podiumsdiskussion zu dem Thema „Mindestlohn“. Der Antwortende, Andreas Clauss, ist Vorstand der Novertis Stiftung ${ }^{24}$ und Geschäftsführer des Schweizer Stiftungsbundes bzw. einer Schweizer Treuhandgesellschaft (wie auf einer Unterseite des Stammtisch München ${ }^{25}$ genannten Internetauftritts zu erfahren ist). Die Novertis ist auf den ersten Blick politisch schwer einzuordnen. $\mathrm{Ob}$ sie einem rechten Lager zugehörig ist, kann man nicht mit Bestimmtheit sagen. Allerdings hören sich die Teaser-Überschriften auf dem Titelblatt der auf Ihrer Homepage umworbenen Zeitschrift Compact ein wenig so an, als könnte sie es sein. Um keine Missverständnisse aufkommen zu lassen: Es geht bei der Analyse des folgenden Beispiels nicht um Fragen der politischen Sympathie oder Antipathie (wobei eher letzteres der Fall wäre), sondern lediglich darum, an einem zweiten Beispiel zu zeigen, dass der schriftsprachliche Zugang zu der so bestimmten $j a-$ aber-Konstruktion auch Bestand für das Gesprochene hat. Das Beispiel selbst ist auf YouTube ${ }^{26}$ zu finden. Für die Analyse hier jetzt ein (sozusagen einfaches, am Text des Gesprochenen orientiertes) Transkript, in dem - wie auch schon bei (26) - wieder Markierungen eingefügt sind:

Beispiele (27)

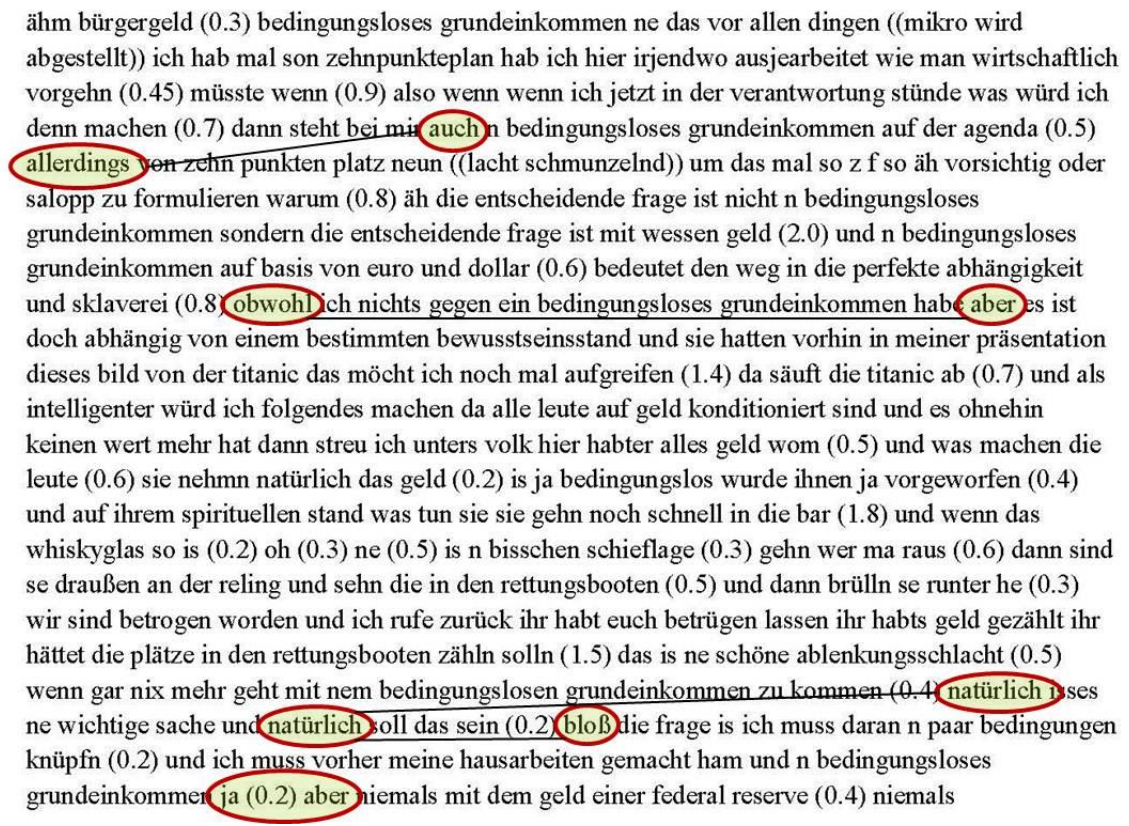

Abbildung 17: Beispiel Mindestlohn (Antwort bei einer Podiumsdiskussion)

\footnotetext{
${ }^{24} \mathrm{Cf}$. http://www.novertis.de. [13.02.2013]

${ }^{25} \mathrm{Cf}$. http://www.stammtisch-muenchen.de/Andreas-Clauss.43149.html\&PHPSESSID=c6da79344bf65b30 $13795 \mathrm{c} 2119 \mathrm{dd} 375 \mathrm{c}$. [13.02.2013]

${ }^{26} \mathrm{http}: / /$ www.youtube.com/watch?v=K21KhiO5xi4. [13.02.2013]
}

ISSN 1615-3014 
Die uns hier interessierende $j a$-aber-Konstruktion ist in $23 \mathrm{zu}$ finden. Es handelt sich um ein Gelenk mit Negation im syntaktisch integrierbaren aber-Teil. Die Funktion wäre demnach: Dafür-dagegen-Splitting. Clauss gibt nach dieser Interpretation hier eine thematisch resümierende Stellungnahme ab: Er ist für ein bedingungsloses Grundeinkommen, aber gegen eine Finanzierung mit den Mitteln einer federal reserve. Dieses Statement wird vorbereitet durch die anderen ja-aber-Konstruktionen: Ein bedingungsloses Grundeinkommen ist auf der Agenda, aber nachrangig (4-5). Gegen ein bedingungsloses Grundeinkommen habe ich nichts, man braucht dafür aber einen gewissen Bewusstseinsstand (9-10). Ein bedingungsloses Grundeinkommen ist eine wichtige Sache, die natürlich sein muss, aber daran müssen ein paar Bedingungen geknüpft werden (20-21).

Das gehäufte Vorkommen dieser Konstruktion macht diese Antwort zu einer voll rhetorisierten und auf Persuasion hin angelegten. Während die ersten drei ja-aber-Konstruktionen (im Kallmeyer'schen Sinn) nach unserer Interpretation Einschränkungen oder Hinzufügungen wären (je nach der Beurteilung der syntaktischen Integrierbarkeit des aber-Teils), handelt es sich bei der letzten um eine Negation. Hier wird ein Schlusswort gesprochen, dessen Hauptelement, die Negation niemals, durch ein wiederholtes finales Vorkommen besonders hervorgehoben wird.

\subsection{Delling vs. Netzer}

Das mittlerweile (oder momentan) nicht mehr als Duo aktive Fußball-Moderatoren-Paar Gerhard Delling (Sportjournalist) und Günter Netzer (ehemaliger Fußballprofi) sind die Protagonisten des letzten Beispiels, das auf YouTube ${ }^{27}$ eingestellt ist und aus dem der hier relevante Ausschnitt als Beispiel (28a/b) transkribiert folgt. Wiederum sind Markierungen eingefügt, um die relevanten Stellen schnell finden zu können. Auf eine prosodische Transkription (mit z. B. Akzenten, Nebenakzenten, Kadenzen, Intonation usw.) wurde ebenfalls wieder verzichtet:

${ }^{27} \mathrm{http} / /$ www.youtube.com/watch?v=cGKjIcD2Wkw. [13.02.2013]

ISSN 1615-3014 
Beispiel (28a)

[4]

\begin{tabular}{|lll|}
\hline Dell hat sich auch nich verändert & $100: 171[00: 17]$ & $00: 18][00: 20]$ \\
\hline
\end{tabular}

[5]

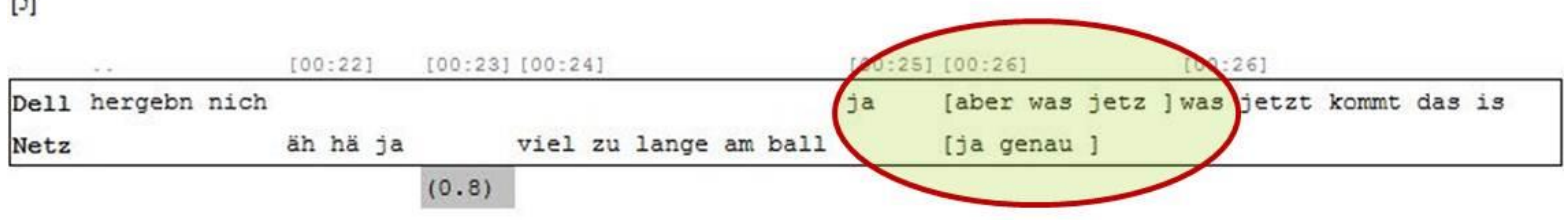

[6]

[00:28] [00:30]

Dell auch (.) bezeichnend also ulli hoeneß kricht den ball nicht ganz weil sie ihn natürlich

[7]

\begin{tabular}{|c|c|c|c|}
\hline & $\ldots$ & {$[00: 32]$} & {$[00: 33]$} \\
\hline $\begin{array}{l}\text { Dell } \\
\text { Netz }\end{array}$ & dementsprechend angespielt habn & 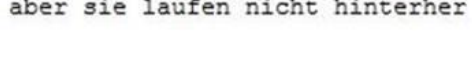 & es gibt bestimmt bessere \\
\hline
\end{tabular}

$[8]$

\begin{tabular}{|lll}
\hline Dell & {$[00: 36]$} & {$[00: 38]$} \\
Netz szenen aus diesem spiel das is doch ad äh f hundertprozentig typisch wieder dass [sie genau sie & {$[i c h$ hab } \\
\hline
\end{tabular}

Abbildung 18: Gerhard Delling (Dell) und Günter Netzer (Netz) I

Diese Interaktion, daran lässt allein schon die Metakommunikation in 8 das is doch wieder hundertprozentig typisch erkennen, reiht sich ein in die Serie ritualisierter Sticheleien, die das Moderatoren-Paar häufig inszeniert. Als ein Mittel zur Stichelei dient auch die ja-aber-Konstruktion in 5. Es handelt sich um eine als TCU auftretende HINZUFÜGUNG, deren Handlungsgehalt also offen ist für die interaktional relevanten Belange. Vorausgegangen ist eine Stelle, an der Delling die Einschätzung abgibt, dass Netzer schon damals den ball nich hergebn wollte. Ein Fehlverhalten, das aber insofern tolerierbar ist, als darin natürlich auch Spielwille zum Ausdruck kommt. Insofern reagiert Netzer erwartungsgemäß, leise ein leichtes Lachen andeutend, mit der Zustimmung viel zu lange am Ball. Mit der ja-aber-Konstruktion, die sich mit einer wiederholten Zustimmung ja genau zunächst überlappt, wird nun das nächste Fehlverhalten thematisch und vorausweisend ausführlich eingeleitet: ja aber was jetzt kommt das is auch bezeichnend, weshalb man den Eindruck bekommen kann, dass diese Stelle nicht zufällig ausgewählt wurde. Dieses Fehlverhalten (nämlich: nach schlechtem Anspiel nicht mehr hinterherlaufen, um zu versuchen, den Ball wieder zu bekommen) hat nichts mehr mit Spielwille, sondern eher etwas mit Faulheit zu tun. Es handelt sich somit nicht mehr ohne Weiteres um ein Fehlverhalten, das scherzhaft vorgeworfen und akzeptiert werden kann. Jedenfalls reagiert Netzer seinerseits mit der Einschätzung, dass die Auswahl genau dieser Stelle wieder hundertprozentig typisch sei und dass sie ausgesucht worden sei, um ihn zu kompromittieren: 
Beispiel (28b)

[9]

$[00: 39$

$[00: 41]$

Dell nicht ausgesucht ] szenen raussuchen die mich da in ähäh irgend die mich kompromittiern das ist

Netz diese ]

[10]

\begin{tabular}{|lcl|l}
\multicolumn{1}{l}{$[00: 43]$} & {$[00: 44]$} \\
\hline Dell wirklich äh & \\
Netz & nein ich hab nur die ballbehauptung gesehn da hab ich gsagt das ist gut den rest
\end{tabular}

[11]

$[00: 48]$

$[00: 50]$

$[00: 51]$

\begin{tabular}{|lll} 
Dell & \\
Netz hab ich ich hab das nich ausgesucht aber ähm (.) es war ein eins zu eins immerhin
\end{tabular}

[12]

[00:53]

Dell es nicht weiter vorzeigen das is wirklich ein ganz schlechtes beispiel

\section{Abbildung 19: Gerhard Delling (Dell) und Günter Netzer (Netz) II}

Die ja-aber-Konstruktion leistet hier eine Relevanzabstufung im Kallmeyer'schen Sinn. Die Zustimmung von Netzer wird heruntergestuft, der folgende Vorwurf eines Fehlverhaltens hochgestuft. Sie steht aber vorrangig im Dienste eines perlokutionären Spiels, dessen perlokutionäre Kraft zur Klasse der Derisativa (= zum Bereich der Scherzhaftigkeit zu zählende emotionale perlokutionäre Kräfte, bei denen Elemente des Spöttischen eine Rolle spielen) gehört und als Frotzeln bezeichnet werden kann (cf. Staffeldt 2007: 192, cf. Günthner 2000: 155-203) - hier illokutionär mit einem Fehlverhaltensvorwurf verbunden. Wie bei allen iocativen perlokutionären Kräften, zu denen auch die Derisativa gehören und bei denen eine scherzhafte Interaktionsmodalität das verbindende Element ist, kann die Situation leicht kippen: „Aus dem Spiel kann schnell bitterer Ernst werden." (Staffeldt 2007: 192). Jedenfalls aber, und das war zu zeigen, ist auch hier das aus dem Zugang über schriftsprachliches Vorkommen von ja aber erwachsene Modell zur Beschreibung der Funktion dieser Konstruktion tragfähig.

\section{Schlussbemerkungen}

Die Analysen haben gezeigt, dass ein schriftsprachlicher Zugang zur Erforschung einer pragmatisch beschreibbaren sprachlichen Einheit wie der ja-aber-Konstruktion, deren konzeptionelle Heimat eher im Gesprochenen vermutet wird, fruchtbar ist. Es können zwei Vorkommensweisen strukturell-funktional voneinander unterschieden werden: das Splitting (als NEGATION oder als EINSCHRÄNKUNG) und die HINZUFÜGUNG.

Das ja-aber-Splitting scheint im Falle der Negation noch am ehesten semantisch kompositional rekonstruiert werden zu können, insofern hier ja im weitesten Sinn responsiv als Zustimmung zu einem Teilaspekt des durch ja aber gesplitteten Oberthemas und aber im weitesten Sinn adversativ als Einleitung einer Ablehnung eines anderen Teilaspektes verstanden werden kann - bei der Einschränkung dreht sich dies um. Nach dem Kriterium der prosodischen Eigenstän- 
digkeit müssten diese Verwendungen von ja aber noch eine mehr oder weniger deutliche Eigenständigkeit von $j a$ aufweisen. Diese Verwendungen hängen aber noch an einem weiteren Kriterium: der syntaktischen Integrierbarkeit des aber-Teils. Gesplittet wird nur, wenn der aber-Teil syntaktisch in das Vorangegangene integriert werden kann (etwa als Angabe im valenziellen Sinn) und nicht eine syntaktisch eigenständige Äußerung ist, die durch aber eingeleitet wird. Die Hauptfunktion des ja-aber-Splittings ist in der Ermöglichung einer Dafür-dagegen-Stellungnahme zu sehen.

Wenn der aber-Teil syntaktisch nicht integrierbar ist, werden Propositionen hinzugefügt. Illokutionär scheinen diese Hinzufügungen nicht festgelegt zu sein. Die solchermaßen durch ja aber angeschlossenen Sprechakte beziehen sich auf vorhergehende, zu denen propositional allerdings keine Adversativität vorliegen muss. Adversativität scheint auf einer anderen Ebene zu liegen, und zwar auf einer pragmatisch beschreibbaren. Ob es sich dabei um die Zurückweisung von Präsuppositionen, um Gegensprechakte oder um Konfrontationen auf der Beziehungsebene handelt, muss im Einzelfall geklärt werden. Das ist mitunter nicht leicht, wie bei einer Stelle in einem Call-Center-Reklamationsgespräch (Beispiel (29)):

In Gespräch 2 [das folgende Beispiel (29) ist ein Ausschnitt daraus; d. Verf.] wendet sich ein älterer Kunde an die Agentin, weil er ein Problem mit einer nicht funktionierenden Rufumleitung auf sein Handy hat. Er schildert sein Anliegen hier bereits zum zweiten Mal und beruft sich auf ein Gespräch, das er mit einer Kollegin von A geführt habe, in dem er sich Schritt für Schritt die Einstellung der Rufumleitung habe erklären lassen. Sein erneuter Anruf sei nötig, weil er bereits am ersten Schritt gescheitert sei. Um die Rufumleitung einzustellen, soll er nämlich bei seinem Festnetztelefon den Hörer abnehmen und das ,Amt' (den Wählton) abwarten.

(Bose et al. 2012: 169)

Beispiel (29)

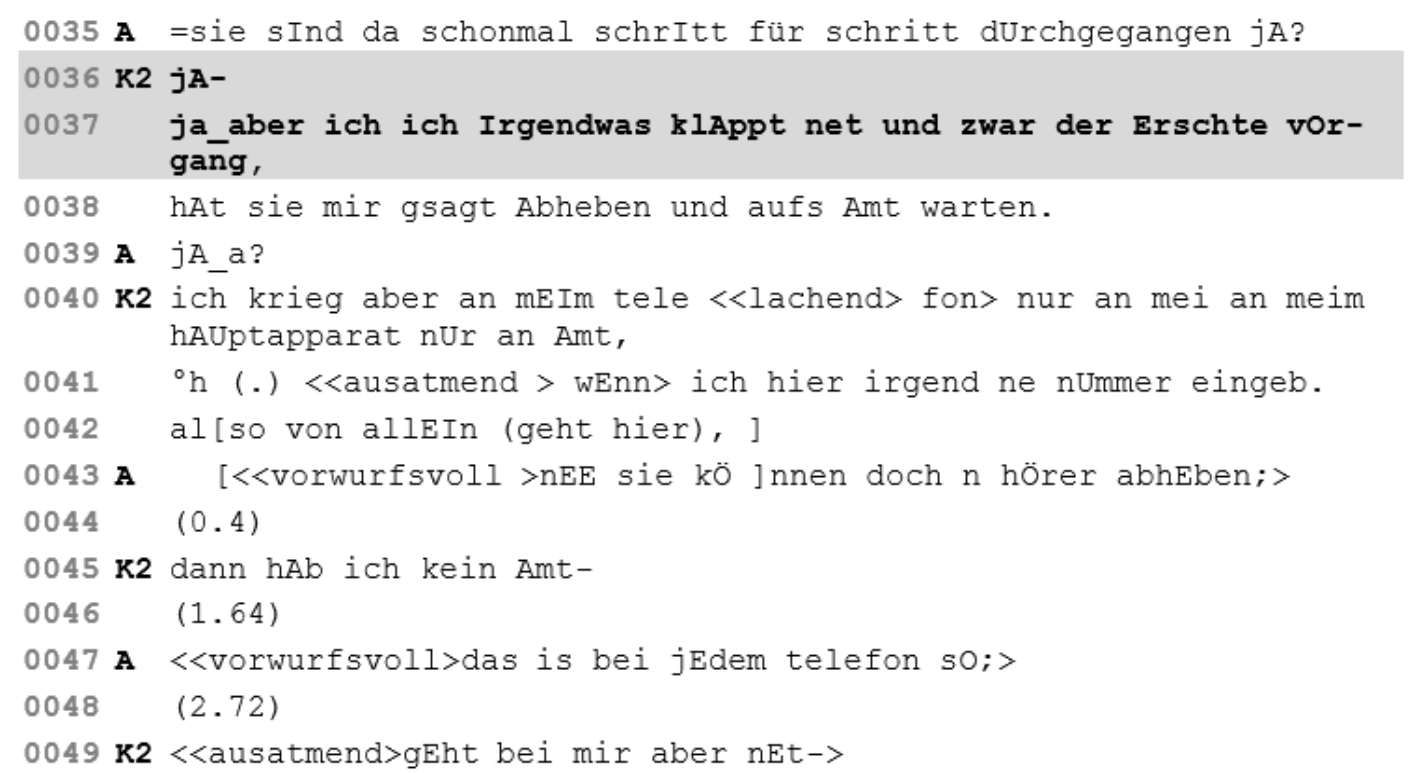

Abbildung 20: Transkriptausschnitt aus Bose et al. 2012: 169 (Hervorhebung durch d. Verf.)

Die ja-aber-Konstruktion (mit prosodischer Anbindung von ja an aber) taucht hier nach einem (prosodisch als eigenständige Äußerung realisiertem) Responsiv-ja auf, mit dem auf eine Frage geantwortet wird. Insofern gibt es keinen Grund, hier von dem Vorliegen eines Koerfer'schen Verstehens-ja auszugehen, denn dass verstanden wurde, wurde bereits klar signalisiert. Eine Präsuppositionszurückweisung könnte hier allerdings vorliegen. Dass man etwas Schritt für 
Schritt schon einmal gemeinsam durchgegangen ist, heißt nämlich nicht, dass es danach dann auch alleine funktioniert. Es könnte sich aber auch einfach um die Angabe des Grundes handeln, warum man ein zweites Mal anruft, obwohl man die Sache beim ersten ja schon Schritt für Schritt durchgegangen ist. Hinzu kommt, dass es sich natürlich auch um einen Zug im (sich im weiteren Verlauf intensivierenden) Beziehungskonflikt mit der Call-Center-Agentin handeln kann, gegen die sich ein Kunde durchsetzen muss, will er nicht als unfähig dastehen, nachdem er ja bereits Schritt für Schritt instruiert wurde. Dafür spräche übrigens dann der Abbruch nach ich ich und der Neustart mit einer unpersönlichen Formulierung, bei der eine Sache nicht funktioniert.

Hinzufügendes ja aber hingegen ist nicht einfach wörtlich ,ja ${ }^{6}+, \mathrm{aber}^{\star}$. Schon deswegen nicht, weil mit ja hier keine noch so sehr verschobene Zustimmung mehr ausgedrückt wird. Weder propositional akzeptierend, noch Verstehen anzeigend, etwas zugestehend, berücksichtigend oder Relevanz teilakzeptierend. Wer diese Konstruktion kompositional verstehen möchte, muss dies pragmatisch-kohäsiv tun, nämlich $j a$ als anknüpfend und aber als gegeninitiativ verstehen. Mit hinzufügendem ja aber nimmt man Bezug auf Vorangegangenes Bezug und formuliert einen Gegenakt, auf welcher Ebene dieser dann auch immer liegen mag.

\section{Literatur}

Ágel, Vilmos/Hennig, Mathilde (2006): „,Theorie des Nähe- und Distanzsprechens“. In: Ágel, Vilmos/Hennig, Mathilde (eds.): Grammatik aus Nähe und Distanz. Theorie und Praxis am Beispiel von Nähetexten 1650-2000. Tübingen, Niemeyer: 3-31.

Aitchison, Jean (1997): Wörter im Kopf. Eine Einführung in das mentale Lexikon. Tübingen: Niemeyer. (= Konzepte der Sprach- und Literaturwissenschaft 56).

Auer, Peter (1997): „Formen und Funktionen der Vor-Vorfeldbesetzung im gesprochenen Deutsch“. In: Schlobinski, Peter (ed.): Syntax des gesprochenen Deutsch. Opladen, WdV: 55-91.

Auer, Peter /Günthner, Susanne (2005): „Die Entstehung von Diskursmarkern im Deutschen Ein Fall von Grammatikalisierung". In: Leuschner, Torsten/Mortelmans, Tanja/De Groodt, Sarah (eds.): Grammatikalisierung im Deutschen. Berlin/New York, de Gruyter: 335-362. http://www.inlist.uni-bayreuth.de/issues/38/Inlist38.pdf.

Barth-Weingarten, Dagmar (2006): ,fuzzy boundaries - Überlegungen zu einer Grammatik der gesprochenen Sprache nach konversationsanalytischen Kriterien“. In: Deppermann, Arnulf/Fiehler, Reinhard/Spranz-Fogasy, Thomas (eds.): Grammatik und Interaktion. Radolfzell, Verlag für Gesprächsforschung: 67-93. www.verlag-gespraechsforschung.de /2006/pdf/gui-barth.pdf.

Blühdorn, Hardarik (2012): Negation im Deutschen. Syntax, Informationsstruktur, Semantik. Tübingen: Narr.

Blumenthal, Andreas (1985): „Scheinalternative, Ja-aber-Taktik, Sokratische Methode - Ansätze zu einer Beschreibung rhetorischer Strategien aus konversationsanalytischer Perspektive“. In: Bausch, Karl-Heinz/Grosse, Siegfried (eds.): Praktische Rhetorik. Beiträge zu ihrer Funktion in der Aus- und Fortbildung. Auswahlbibliographie. Mannheim, Institut für deutsche Sprache: 51-73.

Borbonus, René ( $\left.{ }^{3} 2011\right)$ : Respekt! Wie Sie Ansehen bei Freund und Feind gewinnen. Berlin: Econ. 
Bose, Ines et al (2012): , ${ }^{\circ} \mathrm{hh} \mathrm{hh}{ }^{\circ}$ also von KUNdenfreundlich halt ich da nIcht viel bei ihnen; Analyse und Optimierung von Callcenterkommunikation am Beispiel von telefonischen Reklamationsgesprächen“. Gesprächsforschung - Online-Zeitschrift zur verbalen Interaktion 13/21012: 143-195. http://www.gespraechsforschung-ozs.de/fileadmin/dateien/ heft2012/ag-bose.pdf

Brons-Albert, Ruth (1984): Gesprochenes Standarddeutsch. Telefondialoge. Tübingen: Narr.

Burger, Harald ( ${ }^{4}$ 2010): Phraseologie. Eine Einführung am Beispiel des Deutschen. Berlin: Schmidt.

Deppermann, Arnulf ( $\left.{ }^{4} 2008\right)$ : Gespräche analysieren. Eine Einführung. Wiesbaden: VS.

Deppermann, Arnulf/Helmer, Henrike (2013): „Standard des gesprochenen Deutsch: Begriff, methodische Zugänge und Phänomene aus interaktionslinguistischer Sicht“. In: Hagemann, Jörg/Klein, Wolf Peter/Staffeldt, Sven (eds.): Pragmatischer Standard. Tübingen, Stauffenburg: 111-141.

Dobrovol'skij, Dmitrij (2006): „Reguläre Polysemie und verwandte Erscheinungen“. In: Proost, Kristel/Winkler, Edeltraud (eds.): Von Intentionalität zur Bedeutung konventionalisierter Zeichen. Festschrift für Gisela Harras zum 65. Geburtstag. Tübingen, Narr: 29-64.

Duden ( $\left.{ }^{8} 2009\right)$ : Die Grammatik. Unentbehrlich für richtiges Deutsch. Mannheim: Bibliographisches Institut.

Fillmore, Charles/Kay, Paul/O'Connor, Mary Catherine (1988): "Regularity and idiomaticity in grammatical constructions. The case of let alone". Language 64/3: 501-538.

Fleischer, Wolfgang ( $\left.{ }^{2} 1997\right):$ Phraseologie der deutschen Gegenwartssprache. Tübingen: Niemeyer.

Fleischer, Wolfgang/Barz, Irmhild ( $\left.{ }^{4} 2012\right)$ : Wortbildung der deutschen Gegenwartssprache. Völlig neu bearb. v. Irmhild Barz unter Mitarbeit v. Marianne Schröder. Berlin/Boston: de Gruyter.

Gruber, Helmut (1996): Streitgespräche. Zur Pragmatik einer Diskursform. Opladen: WdV.

Günthner, Susanne (2000): Vorwurfsaktivitäten in der Alltagsinteraktion. Grammatische, prosodische, rhetorisch-stilistische und interaktive Verfahren bei der Konstitution kommunikativer Muster und Gattungen. Tübingen: Niemeyer.

Hagemann, Jörg/Klein, Wolf Peter/Staffeldt, Sven (eds.) (2013): Pragmatischer Standard. Tübingen: Stauffenburg.

Kallmeyer, Werner (2007): „Möglichkeiten der maschinellen Unterstützung bei der Arbeit mit Interaktionskorpora“. In: Kallmeyer, Werner/Zifonun, Gisela (eds.): Sprachkorpora - Datenmengen und Erkenntnisfortschritt. Berlin, de Gruyter: 203-234.

Kallmeyer, Werner (1987): „Bemerkungen zum Verhältnis von Sprache und Interaktion am Beispiel von ,Ja aber““. In: Russ, Charles/Volkmar, Claudia (eds.): Sprache und Gesellschaft in deutschsprachigen Ländern. Beiträge eines Werkstattgesprächs des Goethe-Instituts York in Zusammenarbeit mit dem Department of Language and Linguistic Science der Universität York am 16./.9.85 in York. München, Goethe-Institut: 53-74.

Kallmeyer, Werner (1978): „Fokuswechsel und Fokussierungen als Aktivitäten der Gesprächskonstitution“. In: Meyer-Hermann, Reinhard (ed.): Sprechen - Handeln - Interaktion. Ergebnisse aus Bielefelder Forschungsprojekten zu Texttheorie, Sprechakttheorie und Konversationsanalyse. Tübingen, Niemeyer: 191-241.

Kallmeyer, Werner/Schütze, Fritz (1976): „Konversationsanalyse“. In: Wunderlich, Dieter (ed.): Studium Linguistik. Kronberg/Ts., Scriptor: 1-28. 
Klein, Wolf Peter (2013): „Warum brauchen wir einen klaren Begriff von Standardsprachlichkeit und wie könnte er gefasst werden?“ In: Hagemann, Jörg/Klein, Wolf Peter/Staffeldt, Sven (eds.): Pragmatischer Standard. Tübingen, Stauffenburg: 15-33.

Koerfer, Armin (1979“): „Zur konversationellen Funktion von ja aber. Am Beispiel universitärer Diskurse“. In: Weydt, Harald (ed.): Die Partikeln der deutschen Sprache. Berlin/New York, de Gruyter: 14-29.

Langacker, Ronald W. (1987): Foundations of Cognitive Grammar. Bd. 1: Theoretical Prerequisites. Stanford: Stanford University Press.

Lütten, Jutta (1977): Untersuchungen zur Leistung der Partikeln in der gesprochenen deutschen Sprache. Göppingen: Kümmerle. (Zugl. Phil. Diss. WWU Münster).

Lutzeier, Peter Rolf (1995): Lexikologie. Ein Arbeitsbuch. Tübingen: Stauffenburg.

Meer, Dorothee (2007): ,,,ja er redet nur MÜLL hier.“ - Funktionen von ,ja“ als Diskursmarker in Täglichen Talkshows“. gidi Arbeitspapierreihe 11. http://www.noam.uni-muenster.de/gidi/arbeitspapiere/arbeitspapier11.pdf

Mroczynski, Robert (2012): Grammatikalisierung und Pragmatikalisierung. Zur Herausbildung der Diskursmarker wobei, weil und ja im gesprochenen Deutsch. Tübingen: Narr.

Müller, Andreas (1997): ,Reden ist Chefsache'. Linguistische Studien zu sprachlichen Formen sozialer ,Kontrolle“ in innerbetrieblichen Arbeitsbesprechungen. Tübingen: Narr.

Olbertz-Siitonen, Margarethe (2009): Unterbrechen in zielgerichteten Gesprächen. Eine gesprächsanalytische Untersuchung. Tampere: Tampere University Press. http://tampub. uta.fi/bitstream/handle/10024/66542/978-951-44-7876-5.pdf?sequence=1.

Opalka, Hubertus (1979): untersuchung zu redekonstituierenden funktionen der modalpartikel ,ja'. eine pragmalinguistische grundlagenstudie zur gesprochenen deutschen umgangssprache. Hamburg: Buske. [Anmerkung: Titel und Text in Vollkleinschreibung]

Rolf, Eckard (1983): Sprachliche Informationshandlungen. Göppingen: Kümmerle.

Rolf, Eckard (1997): Illokutionäre Kräfte. Grundbegriffe der Illokutionslogik. Opladen: WdV. Schippan, Thea (2002): Lexikologie der deutschen Gegenwartssprache. Tübingen: Niemeyer.

Schuster, Britt-Marie (2008): „Verarmung oder Bereicherung der Schriftkultur? Zur Beschreibung und Interpretation der Übergangsformen zwischen Parataxe und Hypotaxe im gegenwärtigen Printjournalismus“. Deutsche Sprache 2/2008: 146-176. http://www.ds digital.de/ce/verarmung-oder-bereicherung-der-schriftkultur-zur-beschreibung-und-interpretation-der-uebergangsformen-zwischen-parataxe-und-hypotaxe-im-gegenwaertigenprintjournalismus/detail.html.

Schwitalla, Johannes (2000): „Medienwandel und Reoralisierung. Phasen sprechsprachlicher Nähe und Ferne in der deutschen Sprachgeschichte“. In: Klein, Dorothea (ed.): Vom Mittelalter zur Neuzeit. Festschrift für Horst Brunner. Wiesbaden, Reichert: 669-689.

Schwitalla, Johannes (2002): „Kleine Wörter. Partikeln im Gespräch“. In: Dittmann, Jürgen/Schmidt, Claudia (eds.): Über Wörter. Grundkurs Linguistik. Freiburg i. Br., Rombach: 259-281.

Schwitalla, Johannes (2012): „Syntaktische Zusätze zu abgeschlossenen Sätzen bei Martin Walser“. In: Grucza, Franciszek (ed.): Vielheit und Einheit der Germanistik weltweit. Bd. 4: Sprache in der Literatur. Kontakt und Transfer in der Sprach-und Literaturgeschichte des Mittelalters und der Frühen Neuzeit. Die niederländische Sprachwissenschaft - diachronisch und synchronisch. Frankfurt a. M., Lang: 97-102.

Schwitalla, Johannes ( $\left.{ }^{4} 2012\right)$ : Gesprochenes Deutsch. Eine Einführung. Berlin: Schmidt. 
Spiegel, Carmen (1995): Streit. Eine linguistische Untersuchung verbaler Interaktionen in alltäglichen Zusammenhängen. Tübingen: Narr. [korrigierte, weitgehend seitengleiche Neuauflage 2011. Mannheim: Verlag für Gesprächsforschung.] http://www. verlag-gespraechsforschung.de/2011/pdf/streit.pdf.

Staffeldt, Sven (2007): Perlokutionäre Kräfte. Lexikalisierte Wirkungen sprachlicher Äußerungen im Deutschen. Frankfurt a. M.: Lang.

Staffeldt, Sven (2012a): „(oder) besser gesagt: Funktionen selbstinitiierter Selbstkorrekturen mit besser gesagt in geschriebener Sprache“. In: Hess-Lüttich, Ernest (ed.): Sign Culture Zeichen Kultur. Würzburg, Königshausen \& Neumann: 109-140.

Staffeldt, Sven (2012b): „Wie öffentlich ist die Hand? Über Sinn und Unsinn eines Signifikanztests in der Korpuslinguistik“. Studia Germanistica 11: 101-110.

Staffeldt, Sven (2013): „Auf dem Weg zum pragmatischen Standard mit Entschuldigungen“. In: Hagemann, Jörg/Klein, Wolf Peter/Staffeldt, Sven (eds.): Pragmatischer Standard. Tübingen, Stauffenburg: 85-109.

Staffeldt, Sven (2015): „Einheiten des pragmatischen Standards“. In: Dürscheid, Christa/Schneider, Jan Georg (eds.): Handbuch Satz, Äußerung, Schema. Berlin/Boston, de Gruyter: 326-344. (= HSW 4)

Ziem, Alexander/Lasch, Alexander (2013): Konstruktionsgrammatik. Konzepte und Grundlagen gebrauchsbasierter Ansätze. Berlin/Boston: de Gruyter.

Zifonun, Gisela/Hoffmann, Ludger/Strecker, Bruno (1997): Grammatik der deutschen Sprache. 3 Bände. Berlin/New York: de Gruyter. 